\title{
Research Paper \\ Impact of Intelligent Financial Qualifications on Capital Developing for Iraqi Small and Medium Enterprise
}

Journal of

\section{TANMIYAT AL-} RAFIDAIN

\section{(TANRA)}

A scientific, quarterly, international, open access, and peer-reviewed journal

Vol. 39, No. 127

Sep., 2020

(c) University of Mosul |

College of Administration and Economics, Mosul, Iraq.

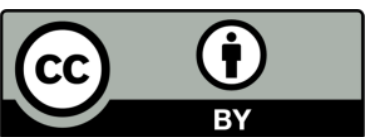

$\boldsymbol{T A N R \boldsymbol { A }}$ retain the copyright of published articles, which is released under a "Creative Commons Attribution License for CC-BY-4.0" enabling the unrestricted use, distribution, and reproduction of an article in any medium, provided that the original work is properly cited.

Citation:Alrawazqee,

Abdulzahra S. and Kadhim, Haider K. , Alaamiree, Ammar S. (2020).“Impact of Intelligent Financial qualifications on Capital Developing for Iraqi Small and Medium Enterprise. TANMIYAT AL-RAFIDAIN, 39 (127), 4969 ,

https://doi.org/10.33899/tanra.20

20.127116.1022

P-ISSN: 1609-591X

e-ISSN: 2664-276X

tanmiyat.mosuljournals.com

\section{Abdulzahra S. Alrawazqee'; Haider K. Kadhim ${ }^{2}$; Ammar S. Alaamiree $^{3}$}

\section{1, $2 \& 3$ General Directorate of Education in Najaf}

Corresponding author: Abdulzahra S. Alrawazqee, aulzahra@yahoo.com

DOI: 10.33899/tanra.2020.127116.1022

Article History: Received: 14/5/2020; Revised: 28/5 /2020; Accepted: 20/7/2020; Published: $1 / 9 / 2020$.

\section{Abstract}

This research aims to analyze the intelligent financial qualifications which can be gain by the financial managers, with its impact to generate new investment opportunities, at Iraqi small and medium enterprises, these enterprises have more risks than other especially under Iraqi economic condition, which improve store market finally. These skills are divided into two groups scientific and occupational, the researchers use an inductive approach for literature review to financial intelligent skills. Research data has been collected by primary data method in some of the academics, enterprise managers, financial managers, accountant, and auditors, at AlNajaf city. after the hypotheses test, the research finds there is a positive impact for financial intelligent skills and capital growth opportunities, and positive relationship between these skills and developing of the securities market, the main recommendation is to open connection channels with international stocks market to integrate local market with them, In addition to the necessity of qualifying financial decision-makers with good financial skills through periodic training courses, facilitating mergers with foreign companies, encouraging managers of small and medium enterprises to enter the stock market and facilitating the procedures required for that.

Keywords:

Financial Intelligence Skills, Capital development, Small and medium enterprises 


\section{ورقة بحثية \\ أثر مؤزهلات الذاكاء المالي على التثمية الرأسمالية للمشاريع

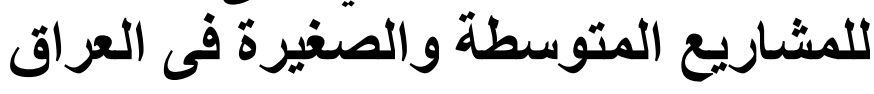

عبد الزهرة سلمان الروازق '؛ حيدركريم كاظم ׳؛ عمار سليم العامري

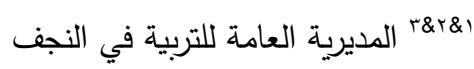

المؤلف المراسل: عبد الزهرة سلمان الروازق، aulzahra@yahoo.com

DOI: 10.33899/tanra.2020.127116.1022

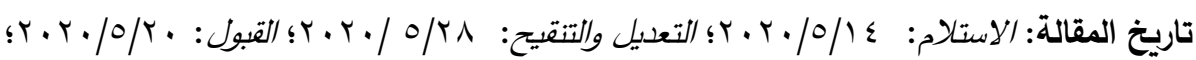

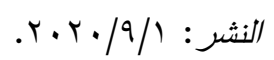

المستخلص

يهذف البحث إلى تحليل أهمية مهارات الذكاء المالي التي يمكن أن ييتلكها متخذي القرارت المالية وتأثيرها

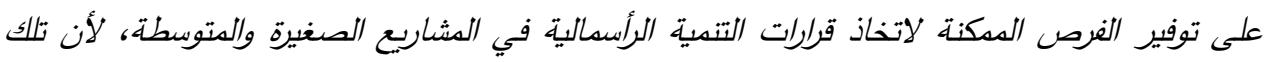

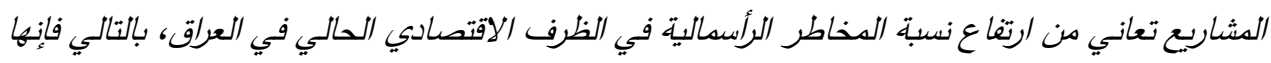

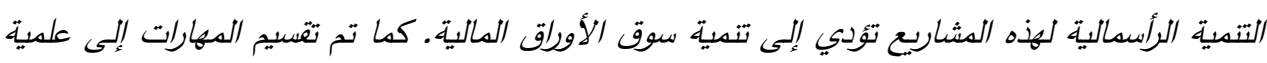

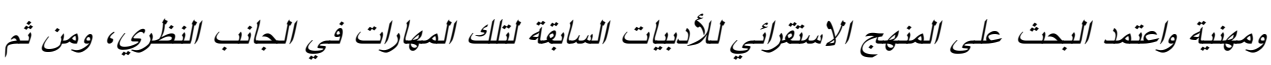

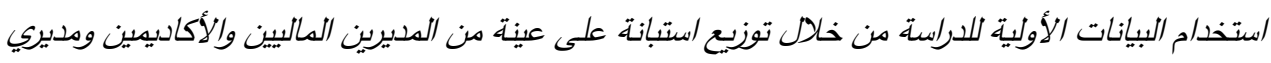

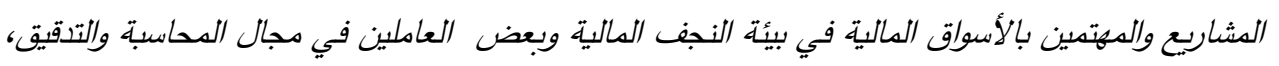

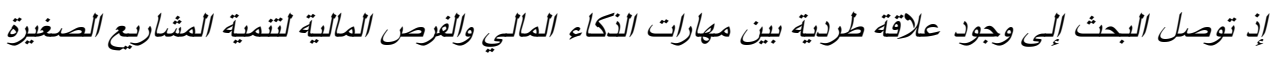

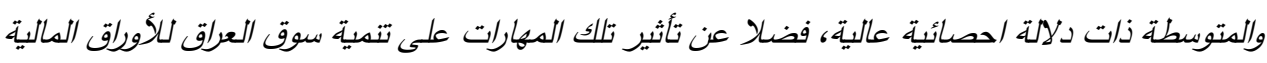

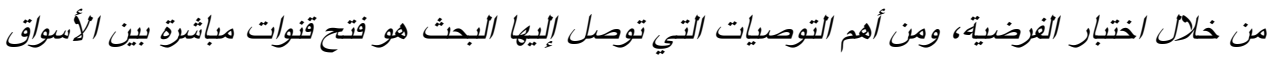

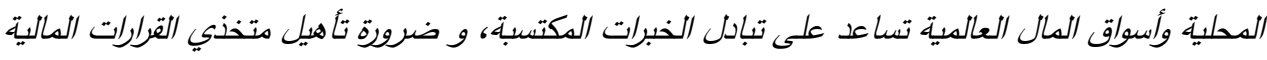

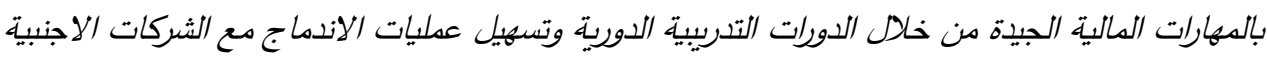

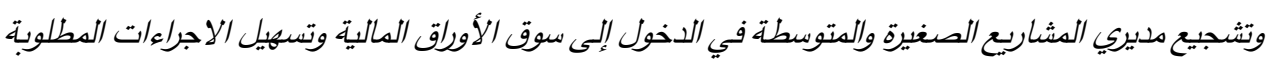

الكلمات المفتاحية

مهارات الذكاء المالي، تنمية رأس المال، المشاريع الصغيرة والمتوسطة

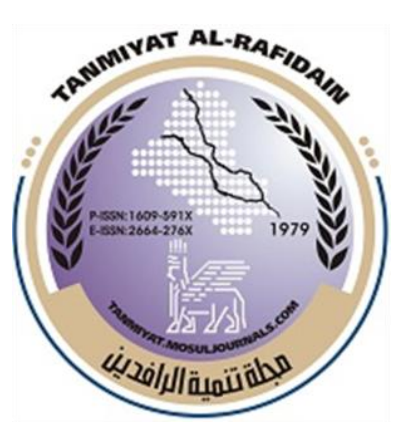

\section{هبلة}

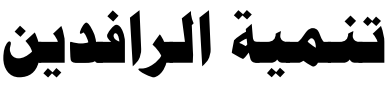

دولة دولية، مفتوحة الوصول، محكمة.

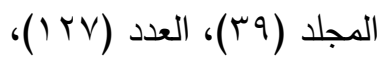

$$
\begin{aligned}
& \text { r.r.r. ايلول r. }
\end{aligned}
$$

ج جامعة الموصل | كلية الإدارة والاقتصاد، الموصل، العراق.

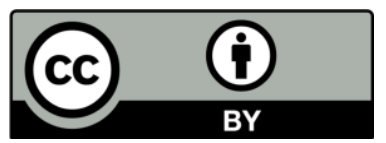

تحتف (TANRA) بحقوق الطبع والنشر للمقالات المنشورة، والتي يتم إصدارها بموجب ترخيص لئن ل (Creative Commons Attribution) (CC-BY-4.0)

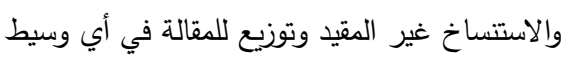
نقل، بشرط اقتباس العمل الأصلي بشكل صحيح.

الاقتباس: الروازق ، عبد الزهرة سلمان و

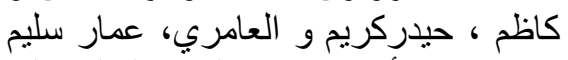

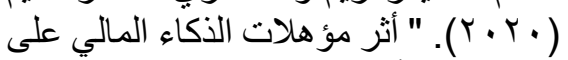

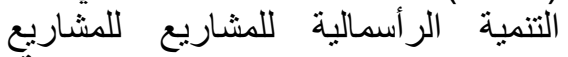
المتوسطة والصغيرة في العراق الت ". تنمبية

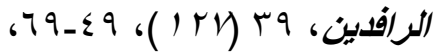
https://doi.org/10.33899/tanra.20 20.127116 .1022

P-ISSN: 1609-591X e-ISSN: 2664-276X tanmiyat.mosuljournals.com 


\section{أثر مؤهلات الذكاء المالي على التنمية الرأسمالية......... الروازق كاظم والعامري}

(1)

(ij)

مقدمة:

تسـى الادارات الصــاعية إلى زيادة نسـبة النمو الرأسـمالي بشـكل مسـتمر لاعمالها ، حيث يتميز

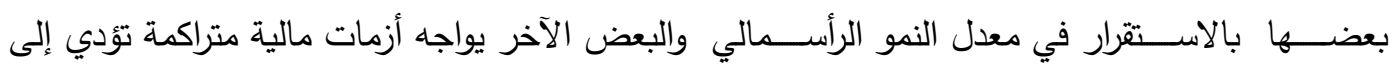

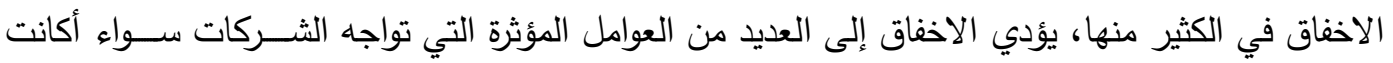

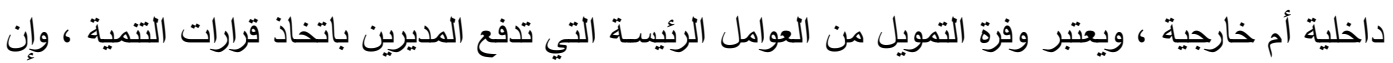

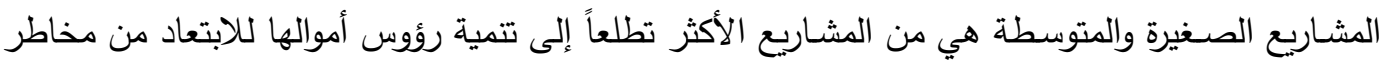
الفشل التتافسي المحيط بأعمال تلك المشاريع.

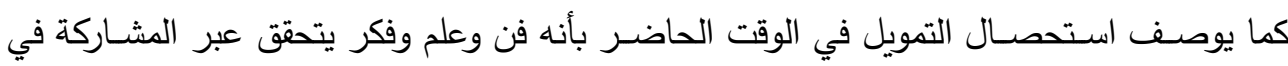

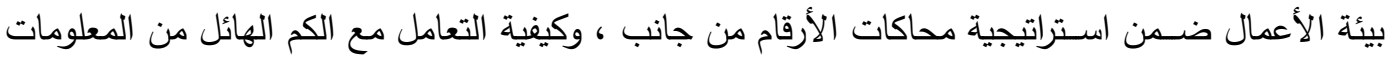

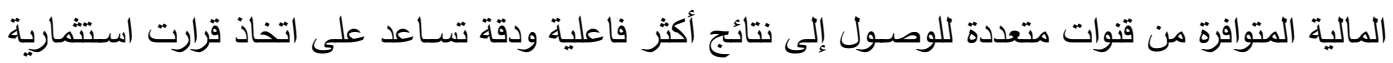

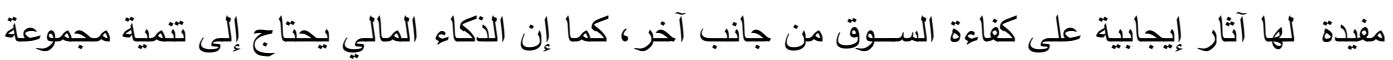

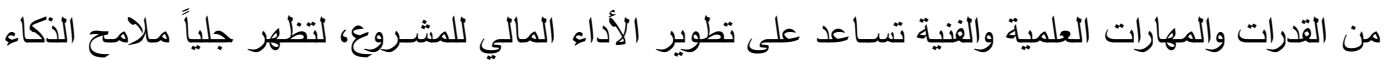

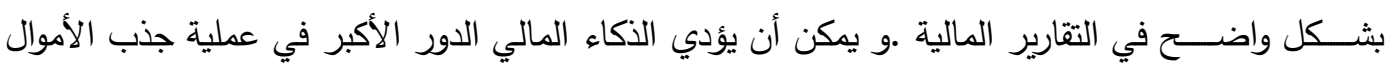
اللازمة التي تتطلبها المشاريع المتوسطة والصغيرة للوصول إلى توسعة عملياتها الانتاجية .

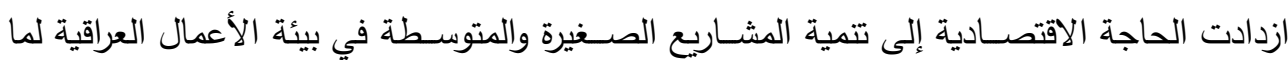
تعانيه من عوامل رافضــــة للتنمية مثل المنافــــة الخارجية وقلة الخبرات المالية وعدم اســـتخدام المعايير المحاسبية بصورة مرضية للمستثمرين والعوامل السياسية. كما تعد المشاريع المتوسطة والصغيرة تعتبر حلقة

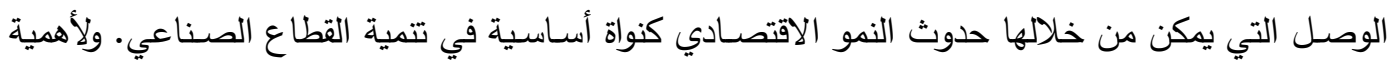
دور مهارات الذكاء المالي في تتمية المشـاريع المتوسـة والصـغيرة ، سـوف يتتاول هذا البحث الدور الذي فئي

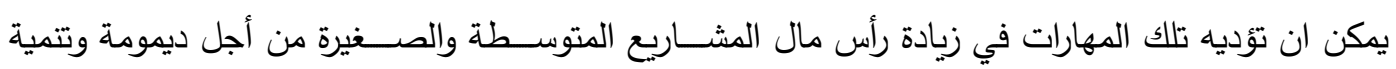
أعمالها انونا منهجية البحث أولاً : مشكلة البحث تتمثل مشكلة البحث بمشـاكل التتمية المالية التي تواجة المشـاريع المتوسطة والصـغيرة بشكل عام وفي البيئة العراقية خصوصاً، إذ إن اكتساب رأس المال يتطلب وجود استقرار مالي ذسبي في سوق الأوراق توات

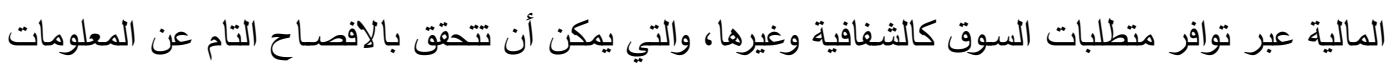
المالية وصولا إلى اتخاذ القرارات المالية بشكل صائب، فضلاً عن طبيعة الأداء المالي الذي يؤديه المديرون

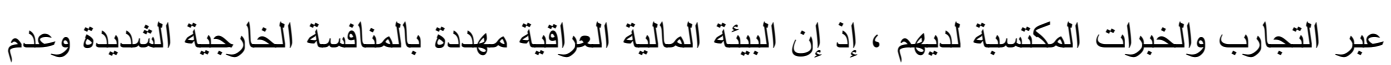
قدرة تلك المشاريع على مجاراتها من أجل الحصول على رأس المال الكافي لتتمية مشاريعهم . 


\section{أثر مؤهلات الذكاء المالي على التنمية الرأسمالية. الروازق كاظم والعامري}

ثانيا: أهمية البحث

تكمن أهمية البحث عبر أهمية مهارات الذكاء المالي المطلوبة لدى متخذي القرارات المالية الناتجة من المهارات المكتسبة والأسس العلمية المستحصلة مسبقا، وإمكانية الاستفادة من تسخير تلك المهارات في مهاري تتمية الأسواق المالية لتلبية الحاجة الرأسمالية للمشاريع الصغيرة والمتوسطة في البيئة العراقية. ثالثا: أهداف البحث يهدف البحث إلى تحليل مدى تأثير مهارات الذكاء المالي على توفير بيئة داعمة للتنمية الرأســمالية

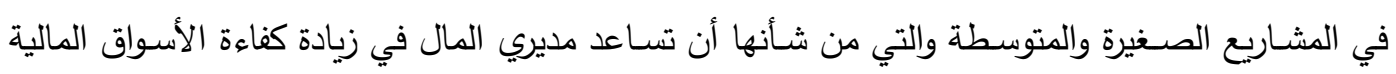
وهي على النحو الآتي :-

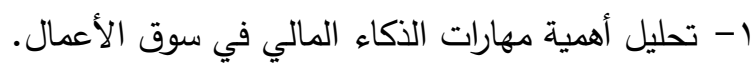
r- تحديد مهارات الذكاء المالي التي يمكن أن يكتسبها متخذو القرارات المالية. r- تحليل أهمية الذكاء المالي في تتمية المشاريع المتوسطة والصغيرة في الأسواق التي تتميز بارتفاع نسبة المخاطر •

ع- تحليل مدى تأثير الذكاء المالي في تتمية أسواق الاوراق المالية. 0- تحديد أهم التحديات التي تواجه نمو المشاريع المتوسطة والصغيرة. رابعا: فرضية البحث

تكمن فرضية البحث في مدى تأثير مهارات الذكاء المالي على تتمية سوق الاوراق المالية، وبالتالي

زيادة الخيارات المتاحة لتتمية رأس مال المشاريع الصغيرة والمتوسطة وكما يأتي :-

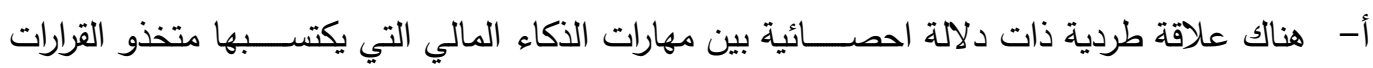

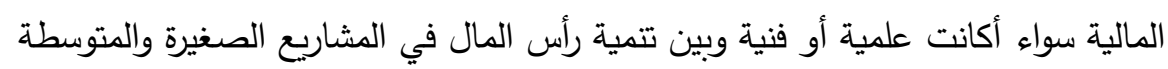

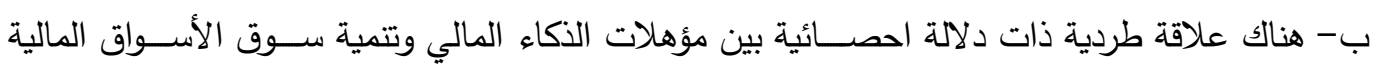
الناشئة .

$$
\text { المبحث الثاني }
$$

أولا: مفهوم الأكاء المالي يتمثل الذكاء المالي بطرائق التفكير التي يسـتخدمها اصـــاب القرارات المالية بالحصـول على الدعم المالي

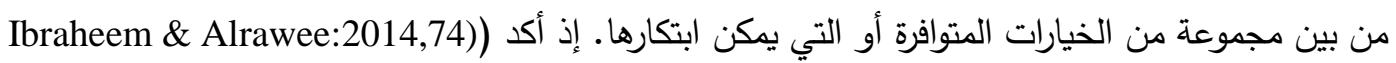

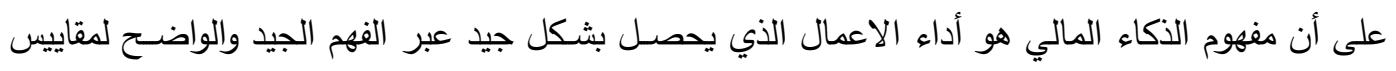

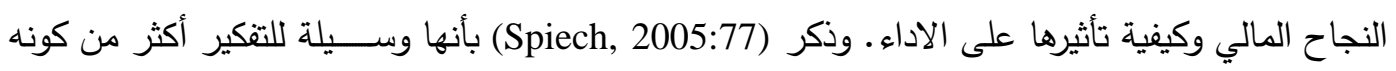
إجراءات متسلسلة متبعة لتحليل أداء الشركة . 
كما عرفها البعض بمهارة قراءة الارقام وقدرات التفكير والتحليل التي تفوق القدرات المكتسـبة والتي تســاعد

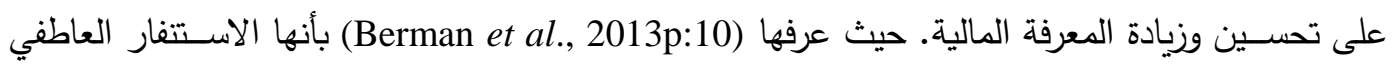
من أجل الحصــول على الحلول المبتكرة التي تعزز من الأداء المالي للثــركة، كما يتطلب هذا الاســتـفار

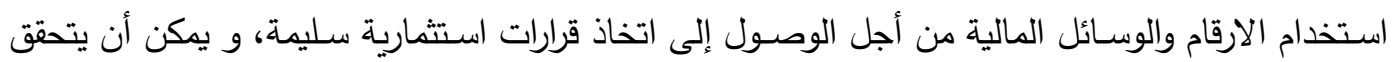

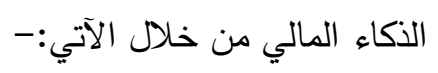

1- فهم أسـس التمويل Understanding Foundation of Finance أي إنها معرفة المقاييس المالية الاساسية عبر قراءة عميقة ومفصلة للقوائم المالية (الميزانية العمومية ، كثف الدخل ، القائمة التدفق

r- براعة التعامل مع القوائم المالية يقصــ بـ التعامل مع الارقام المحاسـبية

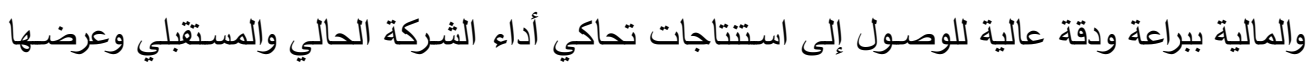

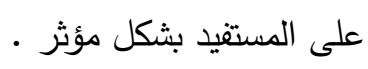
ب- قابلية التحليل understanding of analysis يقصـــ بها إمكانية التحليل المعمق للارقام والمعلومات المالية والمحاسبية وربطها في دالة تساعد على اتخاذ قرارات مالية دقيقة .

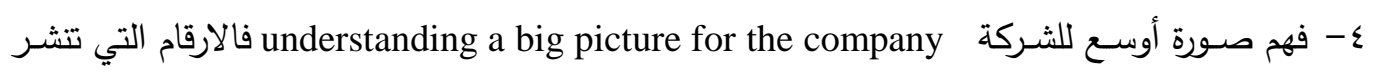
في القوائم المالية لا تكفي لوحدها في الفهم التام للوضـــع المالي في الثــركة ، لذا فإن النتائج المالية

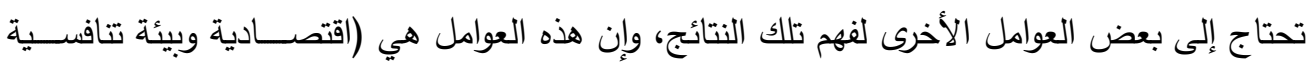

$$
\text { وتكنولوجية وتشريعية وتغيير احتياجات الزبائن). }
$$

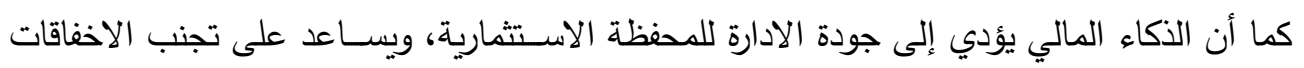
التي تحصل في سوق المال. حيث وضـح (Schutte \& Unlu, 2009:437) بأن الذكاء المالي يساعد على ئى ئل

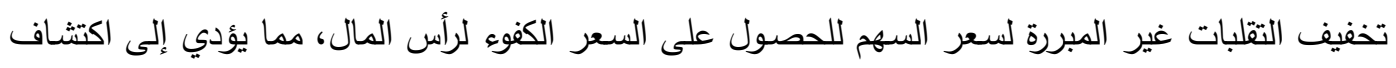

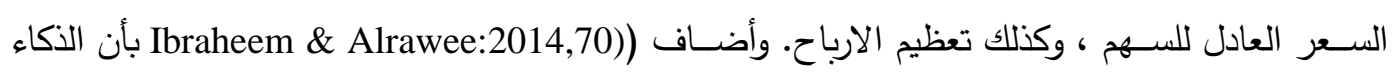

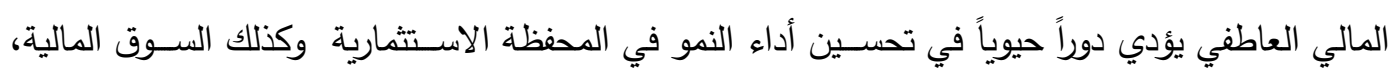

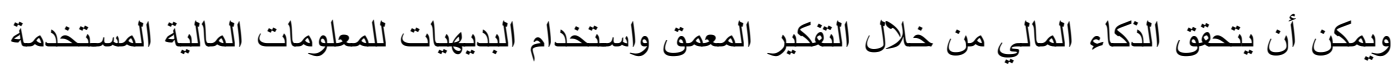

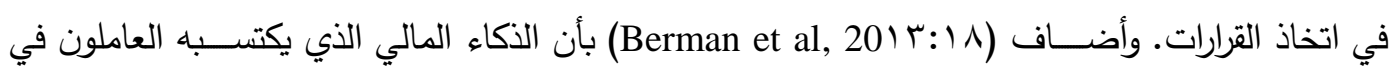
وحدات التحليل يساعد على تحسين أداء الثركة المالي عبر تحليل البيانات والمعلومات المتاحة.

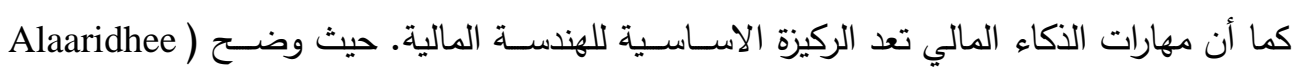

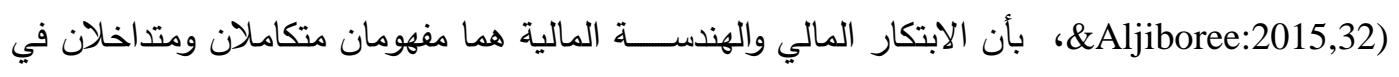
الكثير من المراحل، إذ إن الهندسـة المالية هي أداة تصـــيم تعتمد على الابتكار المالي والذي يعتمد بدوره

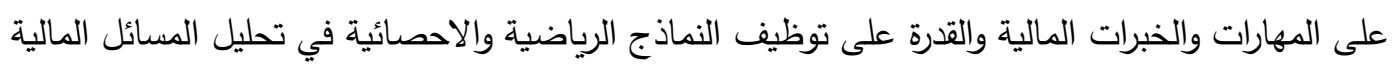
المعقدة للحصول على الابتكارات المالية المطلوبة. 
كما أن التقديم الجيد للقوائم المالية يسـاعد على استدراج المستثمرين لتقديم رأس المال ـ حيث ذكروا

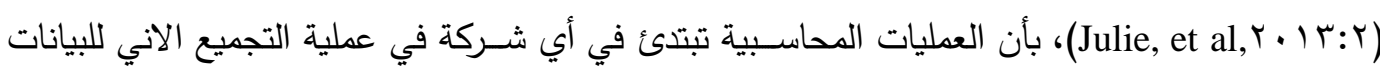

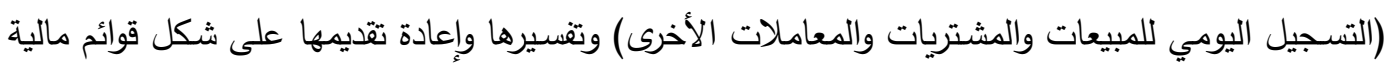

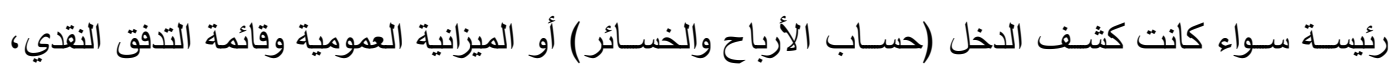

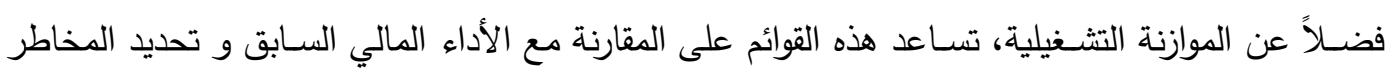

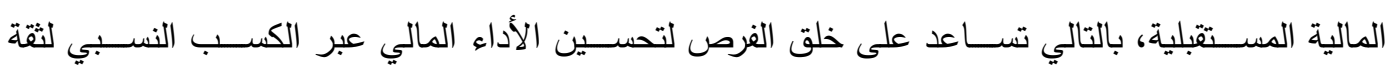
المستثمرين. ثانيا: أهمية الذكاء المالي هناك العديد من الفوائد الرئيســـــة التي يقدمها الذكاء المالي لمتخذي القرارات المالية وهي كما يأتي -: (Berman et al, 201r:11) ו- تساعد على زيادة القدرة بتقييم الشركة بشكل حاسم.

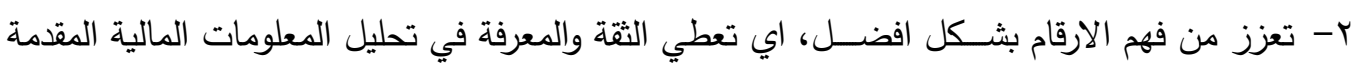
إلى الجهات ذات العلاقة، وتزيد من إمكانية التتبؤات.

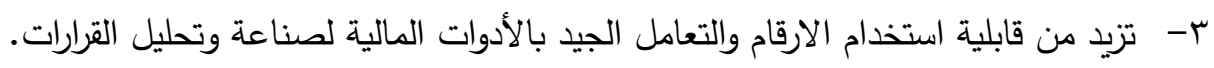

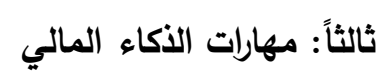

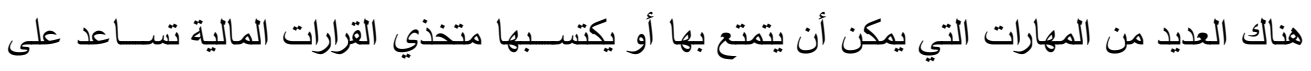

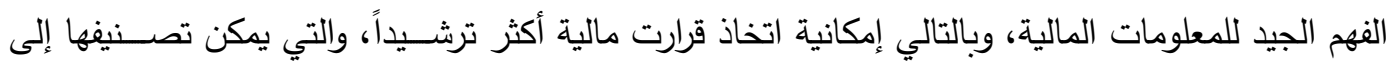

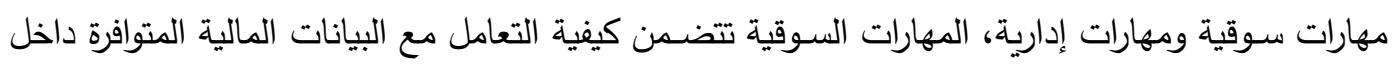

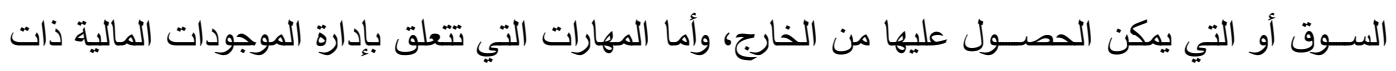
التأثير على تحسين السمعة السوقية للشركة يمكن تسميتها بالمهارات الإدارية. أ-المهارات السوقية

تلك المهارات التي تمكِن صـــناع القرار المالي من شـــراء الموجودات المالية بأقل التكاليف، والتي

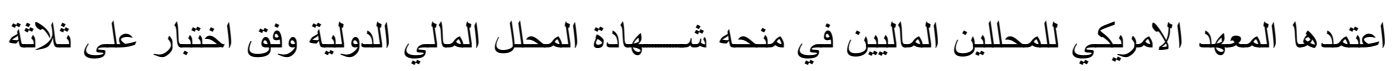

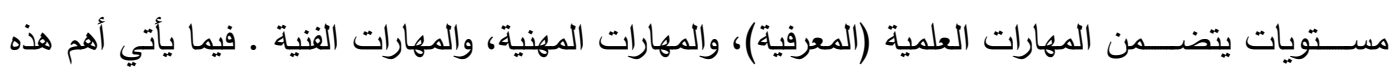

المهارات :- (Ibraheem \& Alrawee:2014,74)

1- القدرة على التتبؤ والاستتتاج في ظل الظروف البيئية المحيطة بالثركة الحالية والمستقبلية .

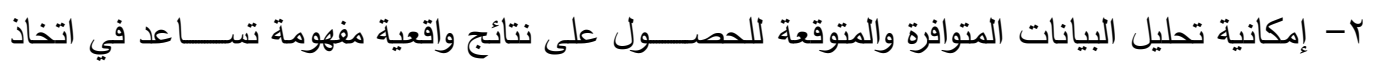
القرارات المالية . ب- التمتع بقدر عالٍ من المسـؤولية تجاه الاخرين ممن يعملون داخل الثــركة أو خارجها وعدم الانحياز لغير مصالح الشركة. 


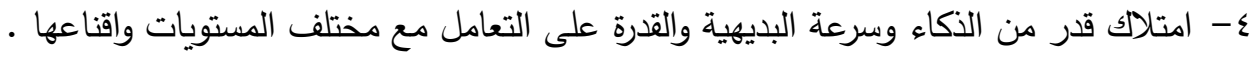

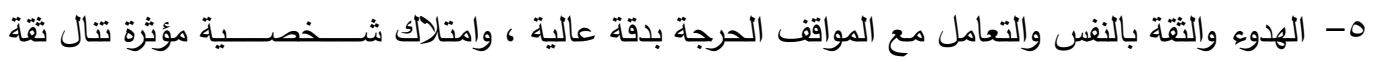

الآخرين.

7- الالمام بالمبادئ والاصول المحاسبية واللوائح التظظيمية ومعرفة ما يصدر منها حديثا وبشكل دوري .

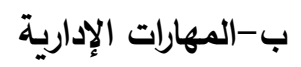

تلك المهارات التي تتمتع بها الادارة المالية ذات العلاقة بالموجودات المالية للثـركة والتي تسـهم في زيادة رغبة المستثمرين لشراء جزء من تلك الموجودات. وفيما يأتي أهم تلك المهارات :- Samkin, et al,

1- مهارات التسعير (pricing), تتضمن تحديد السعر الأمثل للمنتج وصولا إلى أعلى قدر من الأرباح مع

$$
\text { أكثر كمية من المبيعات ضمن ظروف المنافسة الموجودة. }
$$

r- مهارة إدارة حسـابات المدينين والمبيعات الدائنة (managing of debtors and credit sale)، وتثـمل فرجل

$$
\text { الموازنة الدقيقة بين كمية المبيعات على الحساب مع سياسة استحصال تلك الديون. }
$$

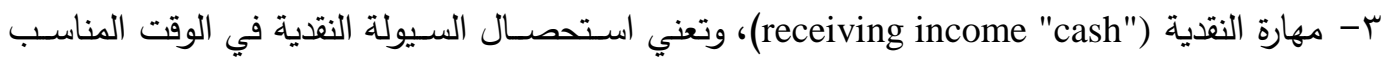

$$
\text { للادارة المالية من العملاء، على الرغم من وجود الكثير من حالات عدم السداد. }
$$

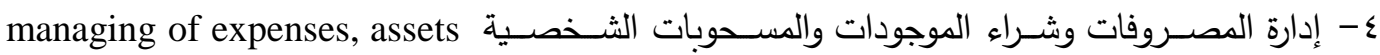
(purchases and drawings) (تلميز هذه المهارة بالأهمية النسـبية في الادارة المالية للمشـاريع، لأن

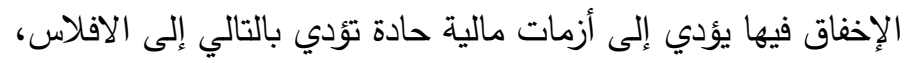

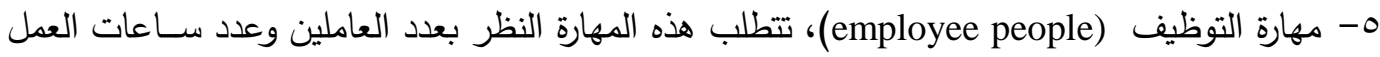

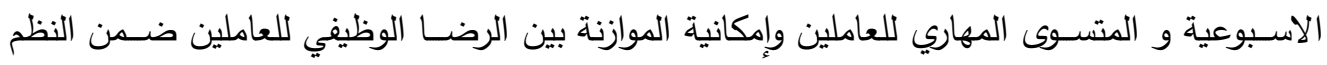

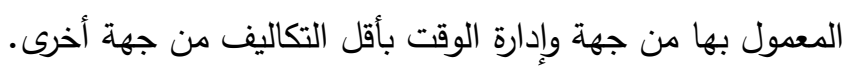

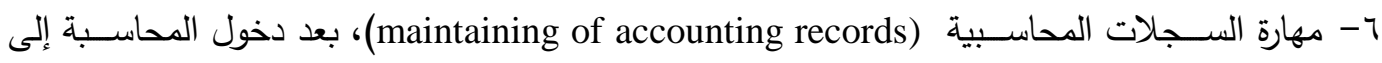
المجال التكنولوجي أصبح تسجيل العمليات المالية يتطلب أساليب مهارية عبر البرامج الذكية التي تقوم

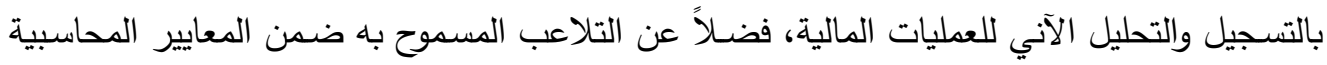
بعناصر القوائم المالية، وهو ما يدعى بالمحاسبة الابداعية (creative accounting).

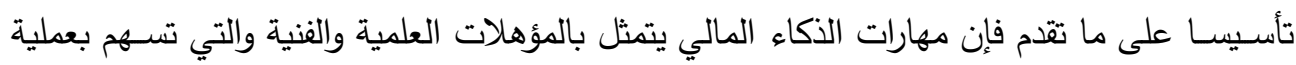

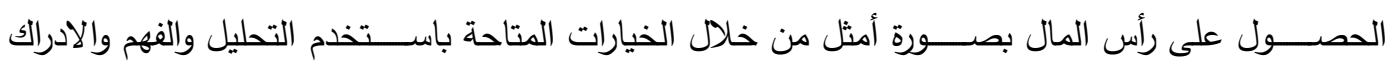
للمعلومات المتاحة في سوق المال، فضلاً عن الإدارة الكفوة للموجودات المالية والتقديم الجيد للقوائم المالية تسهمان في جذب المستثمرين واستدراجهم، كما أن تلك المهارات تعتمد بشكل رئيس على الالمام بالمعرفة المحاسبية وفن التمويل واكتساب الخبرات المهنية عبر الممارسات المستمرة ضمن نطاق السوق والتي تسهم على تتمية كفاءة السوق وكذلك ازدياد كفاءة السوق يسهم بشكل عكسي بتتمية تلك المهارات. انظر الشكل

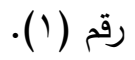




\section{إن الروازق كاظم والعامري}

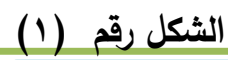

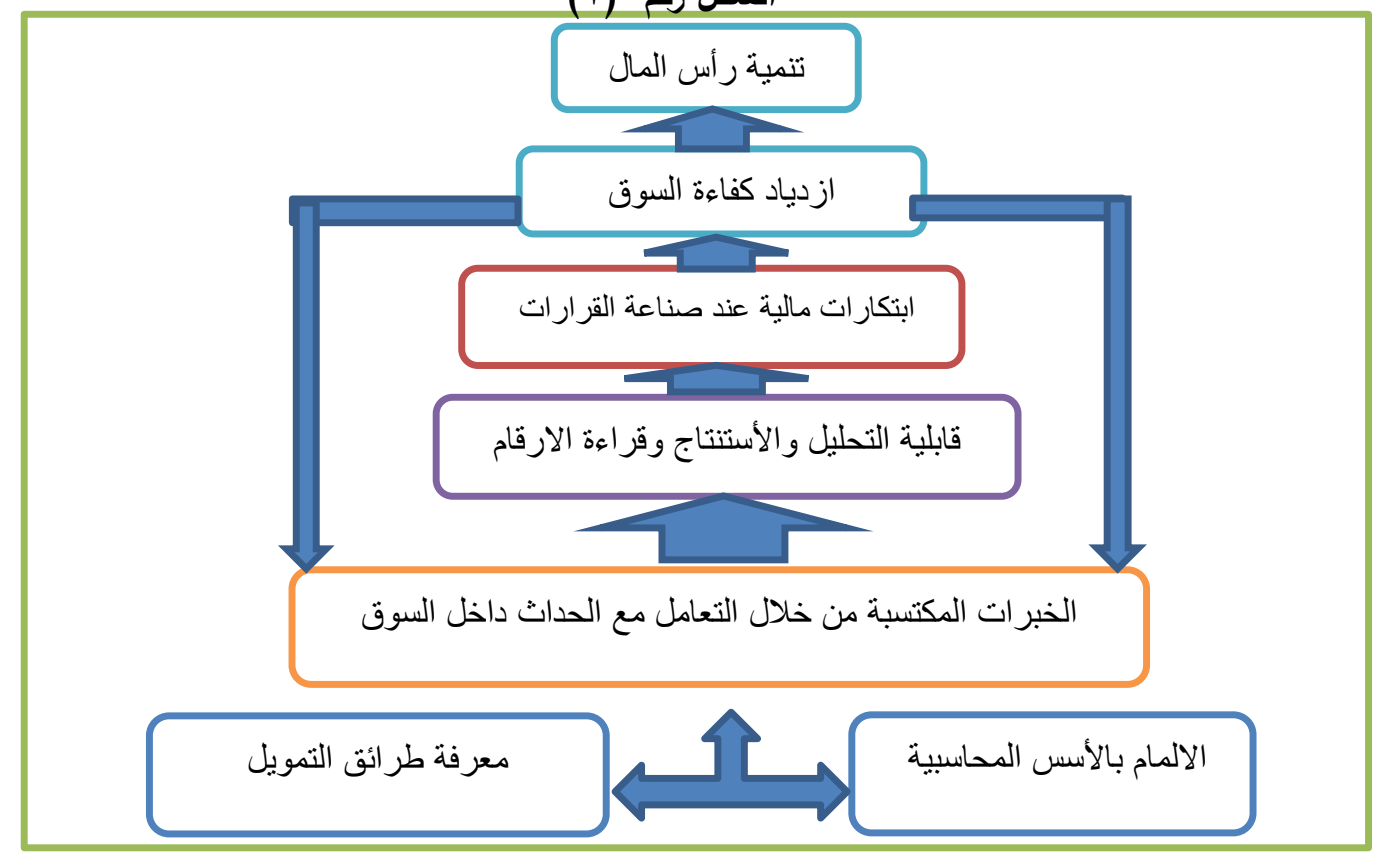

" المصدر : من إعداد الباحثين

المبحث الثاني

التنمية الرأسمالية في المشاريع المتوسطة و الصغيرة

أولا: المشاريع المتوسطة والصغيرة

تصنف المشاريع إلى الصغيرة والمتوسطة والكبيرة، ويعتمد هذا التصنيف على مجموعة من المعايير

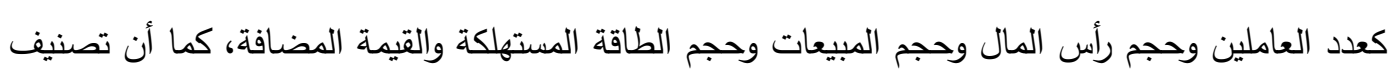
تلك المشاريع يعتمد على قوانين متتوعة في الكثير من البلدان مثل (Sekhree:1995,88). القانون الامريكي والقانون الياباني والقانون الهندي وغيرها

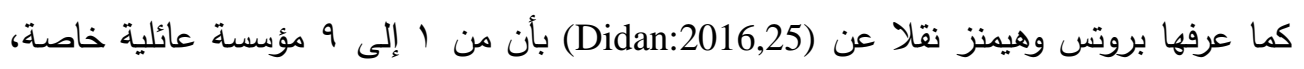

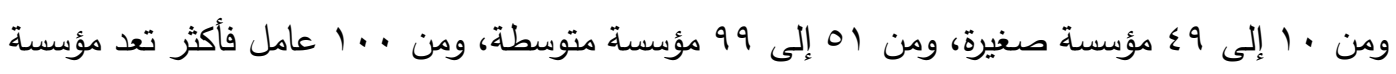

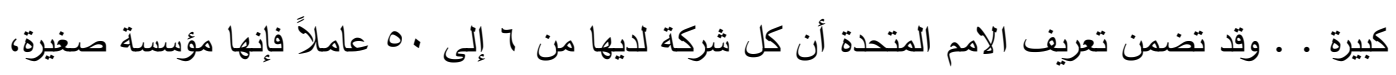

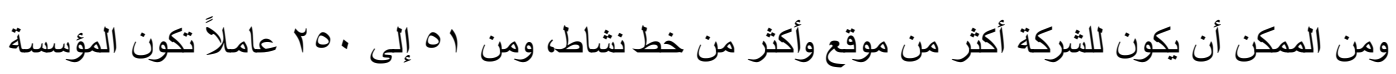

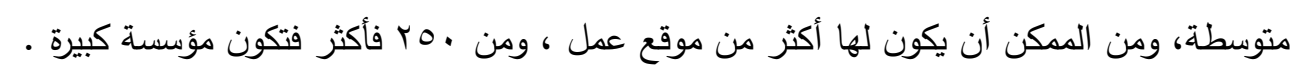

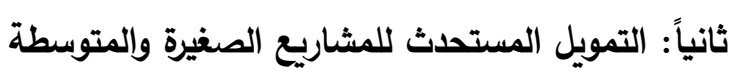

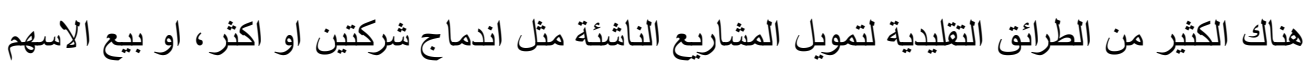
سواءا الاعتيادية او الممتازة ، بيع السندات ، استحصال قروض بمختلف انواعها ، الارباح المحتجزة ، وغيرها. 


\section{أثر مؤهلات الذكاء المالي على التنمية الرأسمالية......... الروازق كاظم والعامري}

الا ان هناك طرق مستحدثة لتمية تلك المشاريع. كما ذكرها (Didan:2016,36)،بان هناك طرق حديثة

للتمويل ظهرت نتيجة البحث المتراكم لتلافي المعوقات التي تجوب طرق التمويل الكلاسيكي ومنها :1- الاستئجار التمويلي ،من خلاله يقوم بنك او مؤسسة ماليه او شركة تفويض المشروع باستخدام الالات

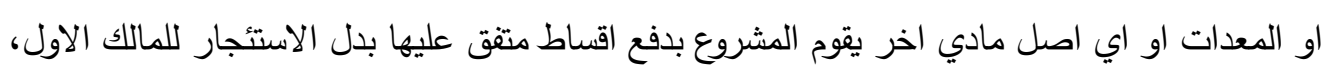

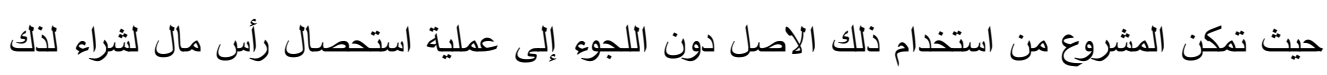

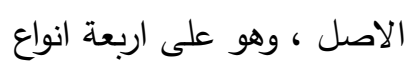

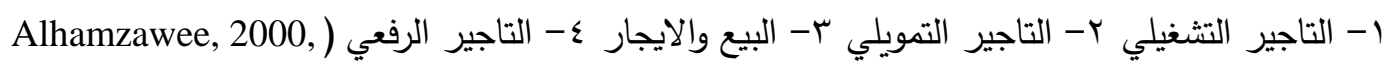

r- التمويل المستحصل من البنوك الاسلامية ، ظهر هذا النوع من التمويل بعد نشوء البنوك الاسلامية

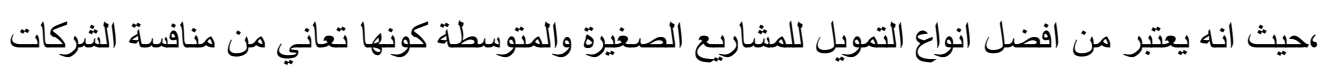
الكبيرة ، تقوم البنوك الاسلامية بتمويل المشاريع المتوسطة والصغيرة على ان تكون لها نسبة من الارباح

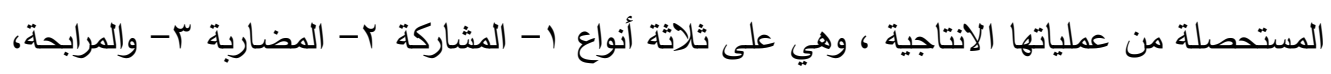

.(Bsharat:2008,68)

r- التمويل الجماعي ،خلال هذا النوع من التمويل يقوم أصحاب الأموال بتجميع الأموال والموارد التي لديهخ

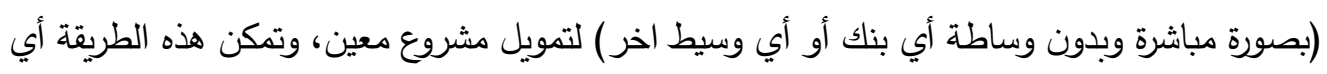

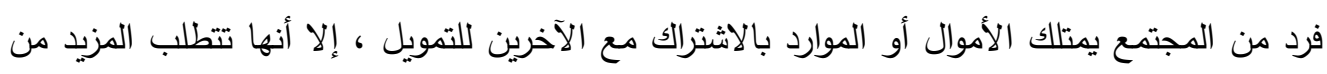
التثة بين المشتركين بالتمويل، (Didan:2016,38). ثالثا: التحديات المالية التي تواجه الشركات الناشئة بالتهوبل تعد قلة الخبرة بالأسواق المالية من أهم المشاكل التي يواجهها رواد الاعمال في المشاريع الصغانه الصغيرة

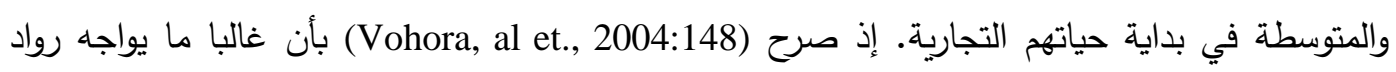

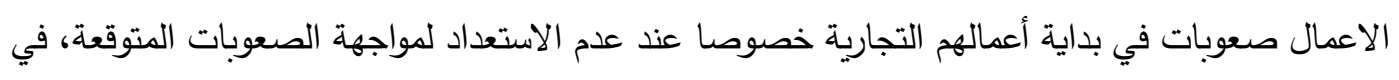
حين إن مشاكل قلة الخبرة في عالم الأسواق المالية تجعلهم يواجهون قلة التدابير المتخذة لتلك الإنة الإمات. ويرى ، أن الوعي في الازمات المستقبلية وقراءة المستقبل بصورة جيدة يساعد (Scott \& Bruce, 1987:440) على تفادي الخسائر المتوقعة وتجاوز تلك الازمات .

كما أن عدم كسب ثقة الجمهور يمثل عقبة كبيرة لدى تلك المشاريع، حيث أشار (Chang, 2015:19) إلى أن الشركات الناشئة تواجه مجموعة من التحديات المالية في بداية دورتها التجارية، من خلال المراحل الاولية لانشاء الثركة و من الضروري الاعتماد على أساس مالي قوي تستطيع الثركة من خلال ممارسة

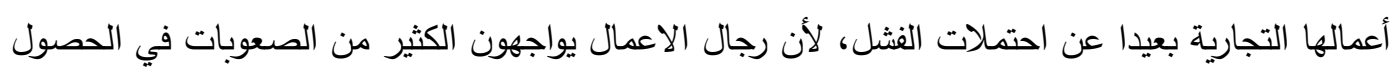

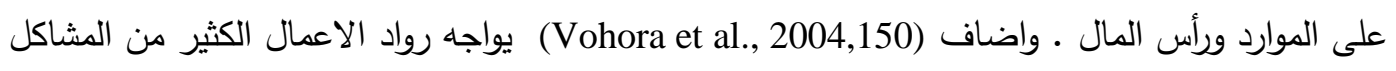

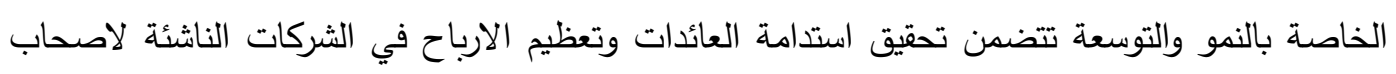




\section{أثر مؤهلات الذكاء المالي على التنمية الرأسمالية. الروازق كاظم والعامري}

رؤوس الاموال. وأكد (Chang, 2015:20) على تثخيص المشاكل التي تواجه تلك الثركات هو عدم حصولها على التمويل من أصحاب رؤوس الأموال إلا بعد الحصول على الاسباب المعقولة للنجاح والاطمئنان

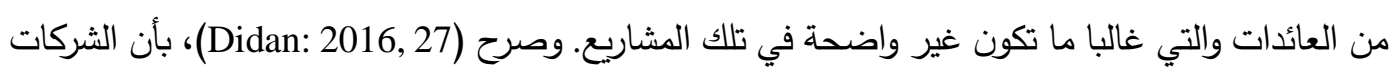

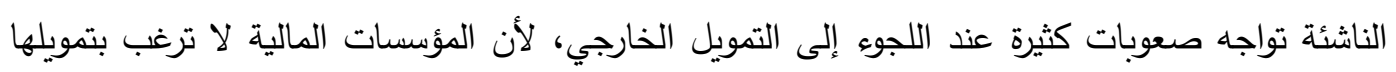

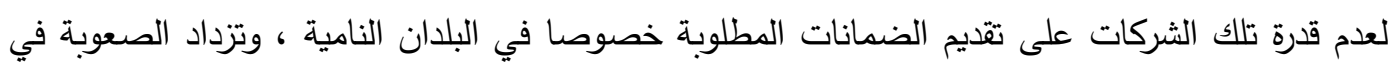
التمويل عند عدم وجود مؤسسات مالية متخصصة في تمويل تلك المشاريع. كما أن شروط الاقتراض التي لتي

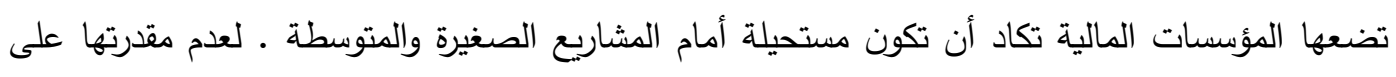
تقديم تلك الضمانات.

كما أن بعض المهارات عند متخذي القرارات للمشاريع المتوسطة والصغيرة تساعد على مواجهة مشاكل

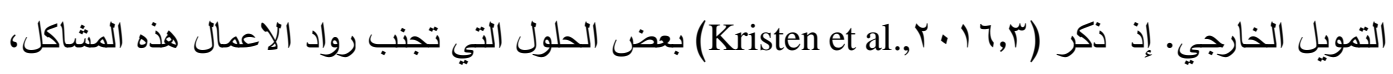
مثل انتاج سلع وخدمات تلبي حاجة المستهلكين تؤدي إلى حصول عوائد مرضية للمستثمرين ، مما يؤدي لوني

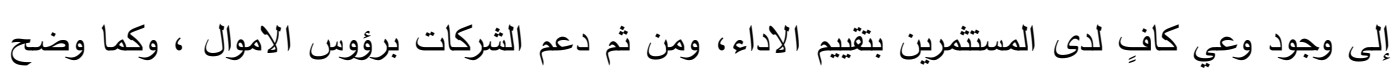

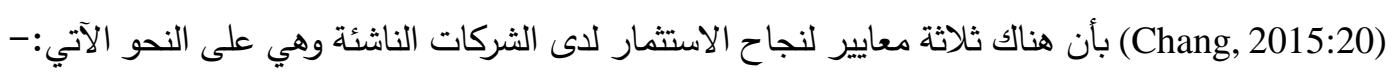

ا - وجود سوق فاعلة تساعد على بيع الاسهم بمجرد اعلانها.

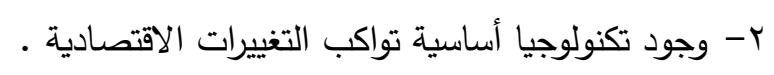
ب- بوجود فريق مؤسس قوي لدى الشركة.

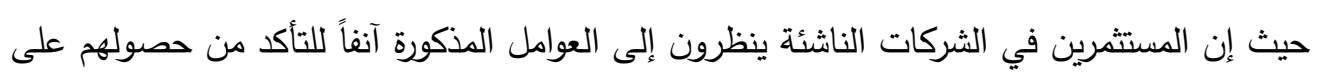
عوائد تتتاسب مع استثماراتهم ، كما أن من الضروري ابتكار طرائق جديدة لجذب المستثمرين ومحاولة طمأنتهم بوجود عوائد كافية لاستثمارهم في الوقت القريب ، وضمان عدم هدر أموالهم في مشروع متهالك.

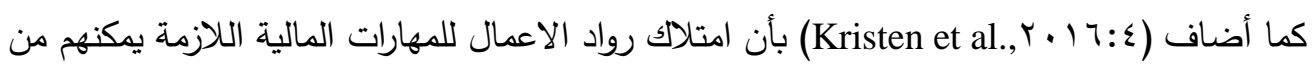
تجاوز تلك التحديات في تتمية أعمالهم الناشئة في مختلف مراحل التطوير .

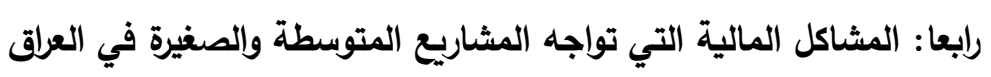

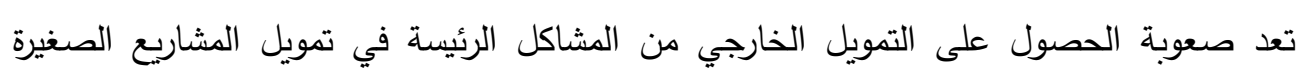

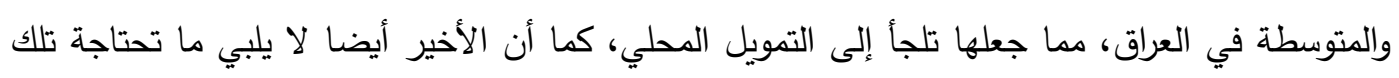

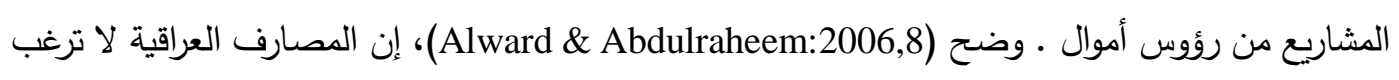
في تمويل تلك المشاريع بسبب عدم المقدرة أو بسبب التقلبات في سعر الصرف للدينار العراقي الحاصلة في

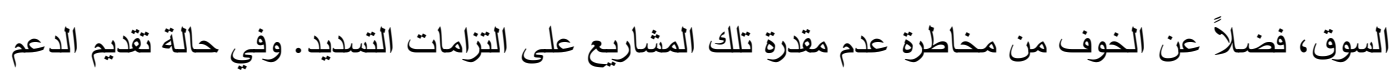

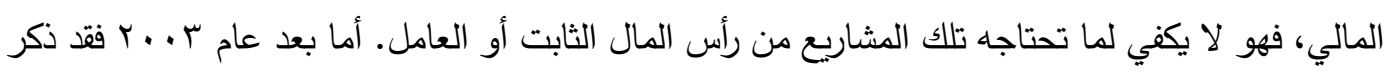

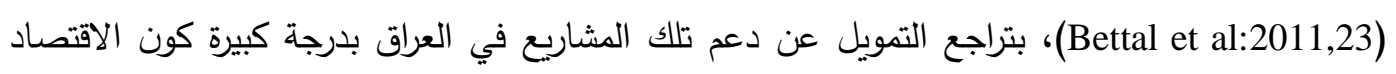

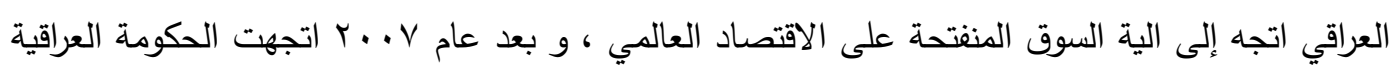


في وضع بعض البرامج لاعم المشاريع المتوسطة والصغيرة عن طريق المصارف المتخصصة من أجل الحد من البطالة وتتمية الواقع الاقتصادي وتوفير بعض مستلزمات المواطنين الاساسية ، لكن تلك البرامج لا تعدو

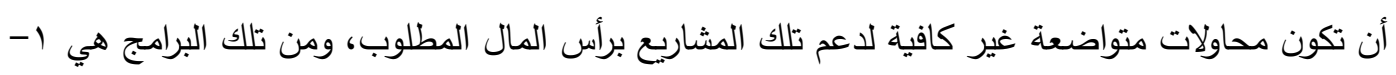

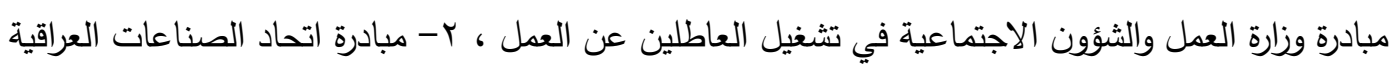

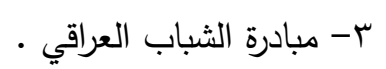
واضاف (Alnasih\& Kamil:2008,164)، بأن المصارف الحكومية العراقية (مصرف الرافدين

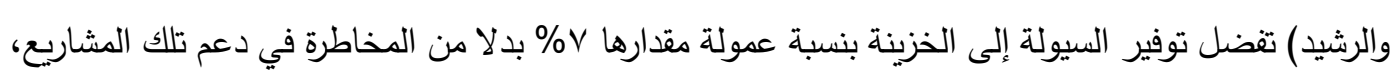

كما أنها ستحمل القروض بفوائد إضافية في حالة الاقتراض لا تستطيع أغلب تلك المشاريع من تسديها. وهناك مشاكل أخرى تواجه تتمية تلك المشاريع ومنها عدم تحديث المعايير المحاسبية المتبعة في البيئة المحاسبية العراقية ، وعدم وضوح الاستراتيجية الاقتصادية العراقية، وعدم كفاءة السوق المالية لبيع أو شراء

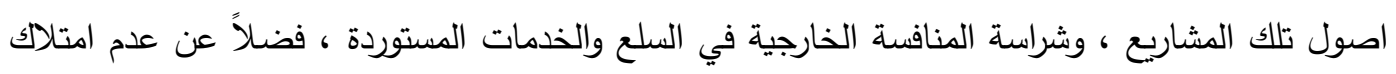
تلك المشاريع من التكنولوجيا الكافية لمجاراة المنافسين • المبحث الثالث الجانب العملي

اعتمد الباحثون على إجراء دراسة استقصائية على عينة من المتخصصين بالثوؤن المالية لقياس مدى العي صحة فرضية البحث، كما أن المعوقات التى تواجه تلك المشاريع في جميع محافظات العراق هي متشابهة

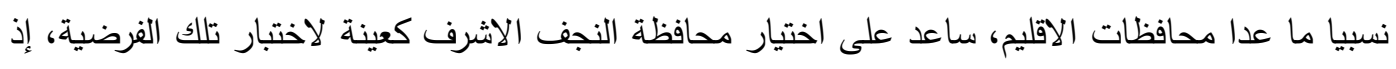

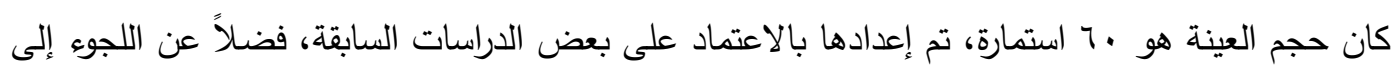
بعض المحكمين في إعداد اسئلة الاستبانة والتي وزعت على مجموعة من الاكاديميين والمديرين الماليين

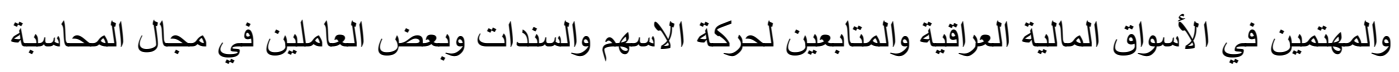

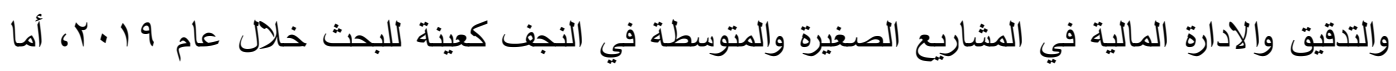
الاستبانات المعادة فهي لـ الدارة المتبانة. مقياس الاستبانة: تم اعتماد مقياس ليكرت في تحليل اجابات الاستبانة وتحويلها إلى نسب وكما مبين في

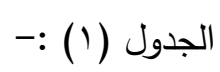

\begin{tabular}{|c|c|c|c|c|}
\hline الاتجاه العام & المرجح & الوزن النسبي & الموافقة & المقياس \\
\hline الفقرة ذات تأثير ودلالة احصائية قليلة جدا & $1,1-1$ & $\% r \cdot-\cdot$ & 1 & لا اتفق بشدة \\
\hline الفقرة ذات تأثير ودلالة احصائية ضعيفة & $r, r-1, \lambda 1$ & $\% \varepsilon \cdot-r_{1}$ & r & لا اتفق \\
\hline الفقرة محايدة ولا يوجد تأثير أو دلالة احصائية & $r, \varepsilon-r, T)$ & $\% ч \cdot-\leqslant 1$ & r & 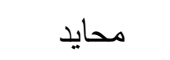 \\
\hline الفقرة ذات تأثير ودلالة إحصائية جيدة & $\varepsilon, r-r, \varepsilon)$ & $\% \wedge \cdot-7$ & $\varepsilon$ & 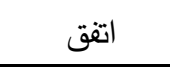 \\
\hline
\end{tabular}




\section{أر. الروازق كاظم والعامري}

\begin{tabular}{|c|c|c|c|c|}
\hline الاتجاه العام & المرجح & الوزن النسبي & الموافقة & المقياس \\
\hline الفقرة ذات تأثير ودلالة احصائية عالية & $0-\varepsilon, Y)$ & $\% 1 \ldots-11$ & 0 & اتفق بشدة \\
\hline
\end{tabular}

ثبات الاستبانة، تم اعتماد مقياس كرونباخ الفا لقياس مدى ثبات اتساق محاور الاستبانة، وقد بين

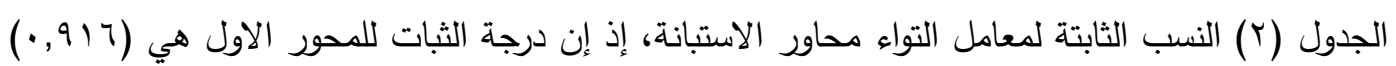

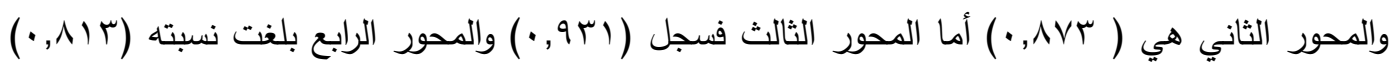

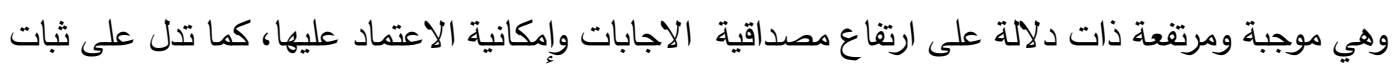
الاجابات في حالة اعادة الاختبار للعينة.

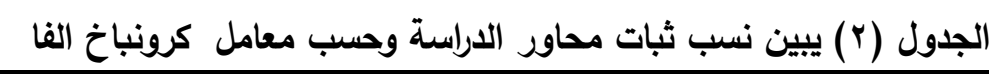

\begin{tabular}{|c|c|c|}
\hline نسب الثبات كرونباخ & محور الارأسة & $ت$ \\
\hline$\cdot, 917$ & مدى ضرورة المهارات العلمية والعملية & 1 \\
\hline$\cdot, \wedge \vee r$ & مدى ضرورة المهارات الفنية & r \\
\hline | & للمشى تأثير الذكاء المالي على زيادة فرص التتمية الرأسمالية & r \\
\hline$\cdot, \wedge)$ & مدى تأثير الذكاء المالي على تتمية الأسواق المالية & $\varepsilon$ \\
\hline
\end{tabular}

$$
\text { أولا: تحليل العوامل الديموغرافية للبحث }
$$

تم التركيز على بيانات المستجوبين ذات التأثير على نتائج البحث ومنها التحصيل العلمي وسنوات التهات الخبرة وكما يأتي:التحصيل العلمي، يبين الجدول ب التحصيل الدرأسي لعينة البحث حيث كانت النسبة الاعلى للحاصلين

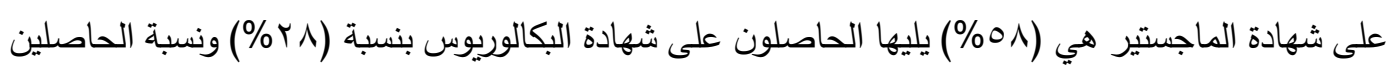

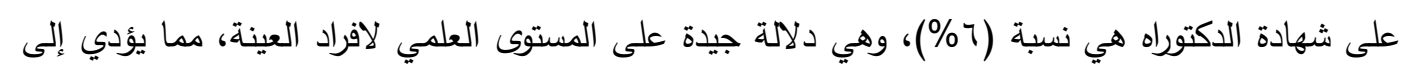
الاطمئنان على دقة وواقعية إجابات الاستبانة.

الجدول (؛ ) التحصيل العلمي لافراد العينة 


\section{أثر مؤهلات الذكاء المالي على التنمية الرأسمالية.........}

\section{الروازق كاظم والعامري}

\begin{tabular}{|c|c|c|c|c|c|}
\hline اعدادية & دبلوم & بكالوريوس & ماجستير & دكتوراه & لتحصيل العلمي \\
\hline r & r & $1 \varepsilon$ & rq & $r$ & التكرار \\
\hline$\% \varepsilon$ & $\% \leq$ & $\%$ rᄉ & $\% \circ \wedge$ & $\% 4$ & النسبة المئوية \\
\hline
\end{tabular}

سنوات الخبرة، يبين الجدول (0) سنوات الخبرة لدى أفراد عينة الدراسة حيث كانت الخبرة الاكثر بين

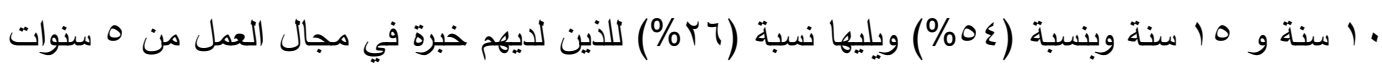

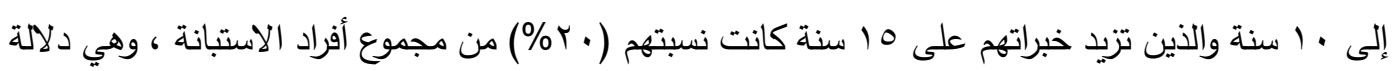

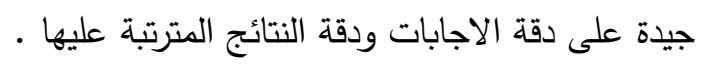
الجدول (•) يبين سنوات الخبرة لعينة الدراسة

\begin{tabular}{|c|c|c|c|}
\hline اكثر من ه 1 سنة & من · ا سنة إلى 1 سنة & من • إلى · 1 سنة & الفترة \\
\hline 1. & rV & r & التكرار \\
\hline$\%$ r. & $\% 0 \leqslant$ & $\%$ \%r & النسبة المئوية \\
\hline
\end{tabular}

ثانيا: تحليل إجابات الاستبانة

المحور الاول - المهارات العلمية لمتخذي القرارات المالية، يبين الجدول في ادناه اجابات الاستبانة

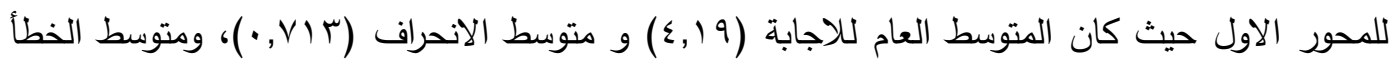

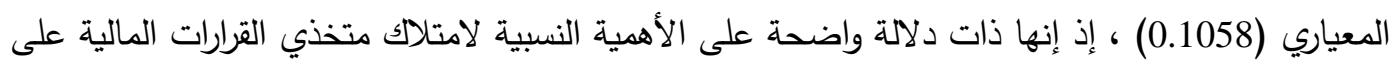

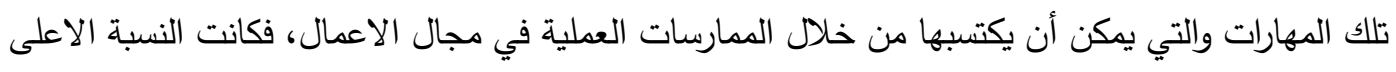

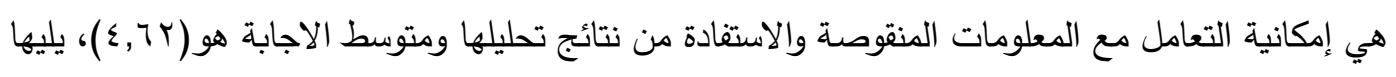

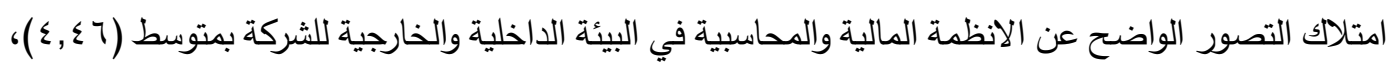

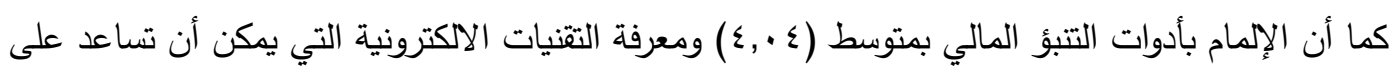

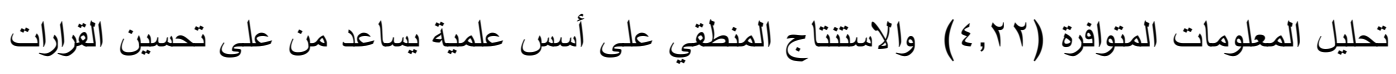

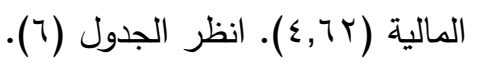

N=50 الجدول (†) المهارات العلمية المطلوبة لمتخذي القرارات المالية 


\begin{tabular}{|c|c|c|c|c|}
\hline متوسط الخطأ & 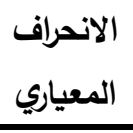 & المتوسط & 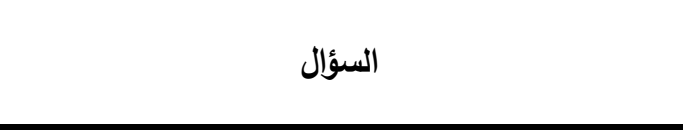 & $ت$ \\
\hline$\cdot, \cdot 9 V$ & $\cdot, T \wedge r$ & 3.94 & المحاسبة في تحليل المعلومات المالية القرارة المالية على توظيف مفاهيم & 1 \\
\hline$\cdot, \cdot \times 1$ & r. & $\varepsilon, \varepsilon\rceil$ & والمحاسبية في بيئة الثركة القرارات تصور عن الانظمة المالية & r \\
\hline$\cdot, \cdot 9$ & $\cdot, 7 \mu \wedge$ & $\varepsilon, \cdot \varepsilon$ & إمكانية إلمام متخذي القرارات بأدوات التتبؤ المالي & r \\
\hline$\cdot, \cdot \vee 9$ & $\cdot, 00 \mathrm{~V}$ & $\varepsilon, \Gamma \varepsilon$ & 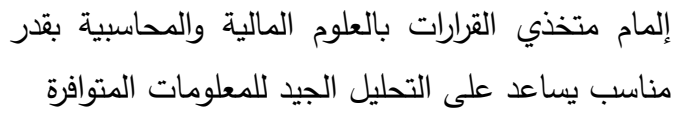 & $\varepsilon$ \\
\hline$\cdot, 119$ & $\cdot, \wedge \varepsilon \cdot$ & $\varepsilon, r, r$ & المحاسبية في الكومبيتر إمام القرار المالي باستخدام البرامج & $\circ$ \\
\hline$\cdot, 19 \leq$ & $\cdot, 7 \Upsilon \varepsilon$ & $\varepsilon, Y_{\uparrow}$ & الاستثمار بشكل مستمر متخذي القرارات على لوائح وقوانين & 1 \\
\hline$\cdot, 11 \leq$ & $\cdot, \wedge \cdot \wedge$ & $\varepsilon, \cdots$ & حالات التنبؤنية متخذي القرار على توظيف الخبرات العملية في & $\checkmark$ \\
\hline 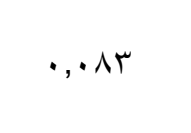 & $\cdot, 019$ & $\varepsilon, \cdot r$ & أداء الشانية متخذي القرار بوضع مؤشرات تحليلية محايدة عن & 8 \\
\hline , , . & $\cdot, 9 \leqslant 0$ & $\varepsilon, 7 r$ & من خلال الاستنتاج المنظي القرار من التعامل مع البيانات المنقوصة & 9 \\
\hline$\cdot, 1 Y \wedge$ & $\cdot, 9 \cdot \varepsilon$ & $\varepsilon, \cdots$ & في ربط متخذي القرار المالي باعتماد قواعد السلوك المهني & 10 \\
\hline 0.1058 & $\cdot, \mathrm{v} \cap \mathrm{r}$ & $\varepsilon, 19$ & المتوسط & \\
\hline
\end{tabular}

المحور الثاني- المهارات الفنية لمتخذي القرارات المالية

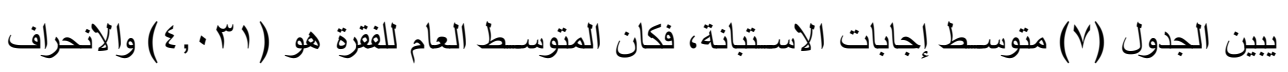

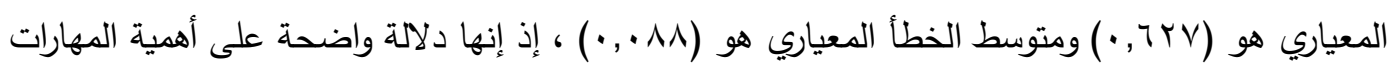

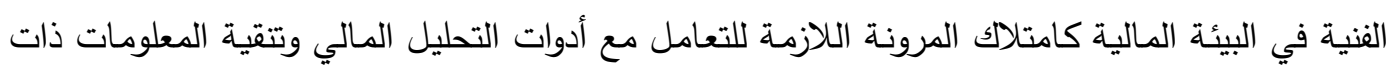

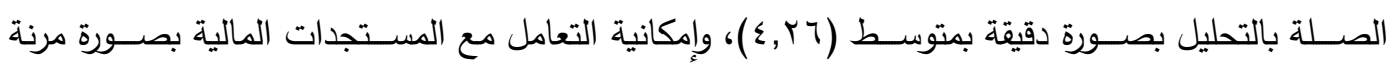

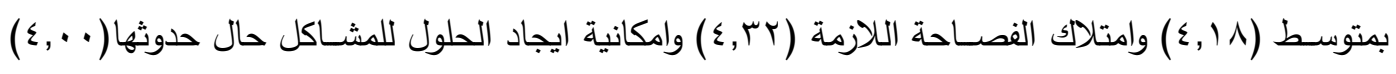

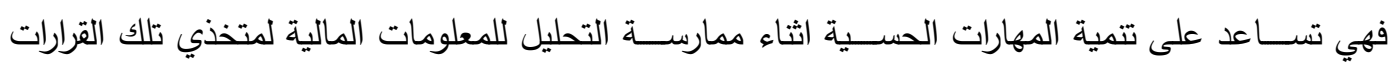
وكذلك امكانية تتمية تلك المهارات والتي تعزز من محاولات الحصول على رأس المال.

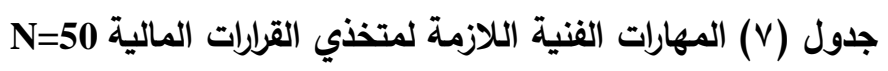




\section{أثر مؤهلات الذكاء المالي على التنمية الرأسمالية......... الروازق كاظم والعامري}

\begin{tabular}{|c|c|c|c|c|}
\hline متوسط الخطأ المعياري & الانحراف المعياري & المتوسط & السؤال & ت \\
\hline תז •, & $\cdot, \varepsilon \leqslant \Gamma$ & $\varepsilon, Y \uparrow$ & ضلازمة للتعامل مع ادورة امتلاك متخذي الترارات المرونـة التحليل المناسبة & 1 \\
\hline$\cdot, \cdot \wedge \wedge$ & $\cdot, 7 Y)$ & $\varepsilon, T_{T}$ & 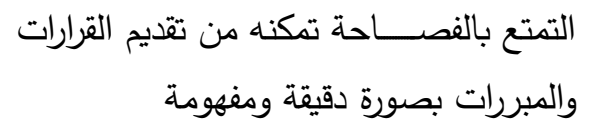 & r \\
\hline$\cdot, \cdot V V$ & $\cdot, 0 \leq \leqslant$ & $\varepsilon, \mu$. & 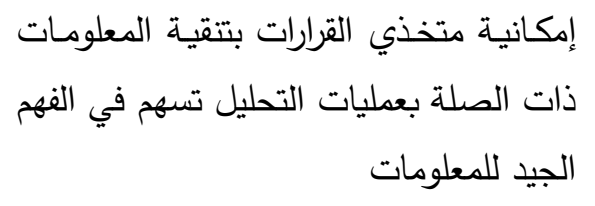 & $r$ \\
\hline$\cdot, \cdot \vee \leqslant$ & $\cdot, O Y M$ & $\varepsilon, 11$ & 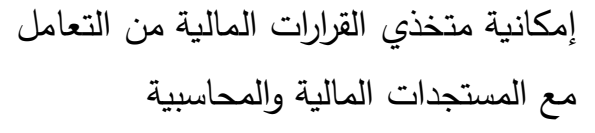 & $\varepsilon$ \\
\hline$\cdot, 10 r$ & $1, \cdot v_{0}$ & r, r & 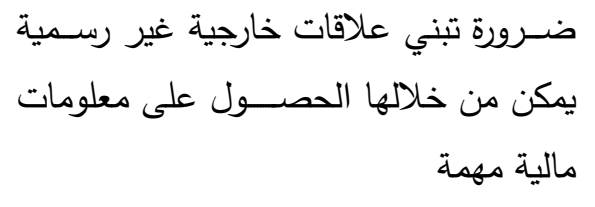 & 0 \\
\hline$\cdot, \cdot 9 r$ & •, Tor & $r, q \leq$ & 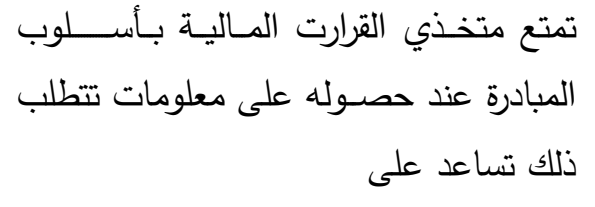 & 7 \\
\hline$\cdot, \cdot \vee V 7$ & $\cdot$, oro & $\varepsilon, \cdots$ & 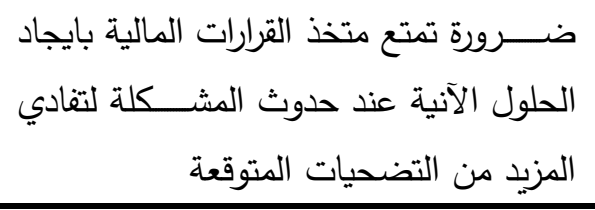 & $v$ \\
\hline 0.088 & $\cdot, T Y V$ & $\varepsilon, \cdot M$ & المتوسط العام & \\
\hline
\end{tabular}

المحور الثالث-مدى تأثير مؤهلات الأكاء المالي على التنمية الزأســــالية في المثـــاريع الصـــيرة والمتوسطة

يظهر الجدول (^) نســـبة اجابات الدراســـة حول محور تأثير مؤهلات الذكاء المالي على التتمية

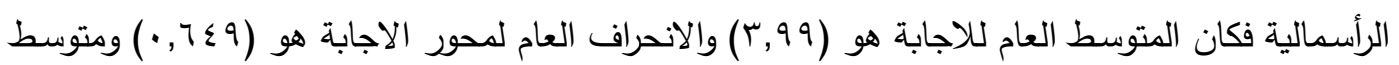

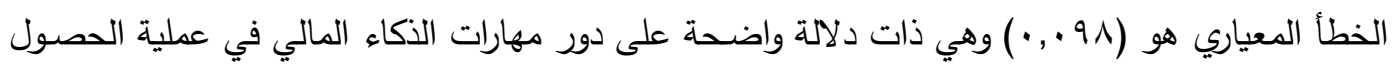

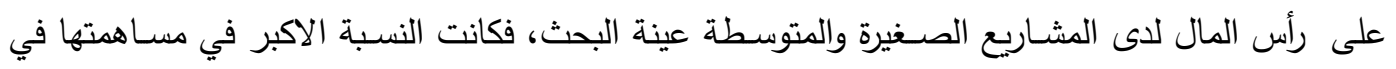

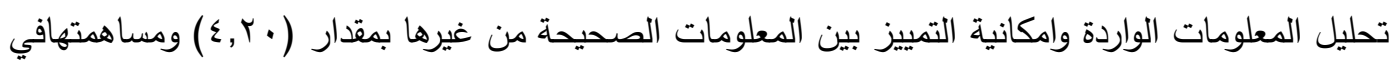

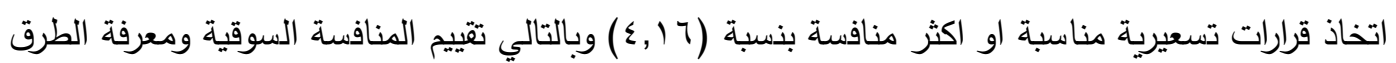

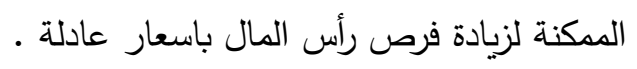




\section{أثر مؤهلات الذكاء المالي على التنمية الرأسمالية. الروازق كاظم والعامري}

N=50 الجدول (^) مدى تأثير المهارات المالية على زيادة فرص التمية الرأسمالية للمشاريع الصغيرة والمتوسطة

\begin{tabular}{|c|c|c|c|c|}
\hline متوسط الخطأ & المعياري - الاف & المتوسط & السؤال & $ت$ \\
\hline$\cdot, \cdot 11$ & $\cdot, 0 \mathrm{~V}$. & $\varepsilon, \cdot \varepsilon$ & تساعد متخذي القرارات المالية على تقييم & 1 \\
\hline$\cdot, \cdot V r$ &., 010 & $\varepsilon, \cdot \varepsilon$ & تمكن متخذـي القرار المـالي من قراءة امكانيـة & r \\
\hline$\cdot, 1 \cdot r$ & •, VYT & $r, v \varepsilon$ & تحقز متخـذي القرارات المـاليـة على تحـدي & $r$ \\
\hline$\cdot, .9 V$ & $\cdot, 719$ & $\varepsilon, 1 \varepsilon$ & 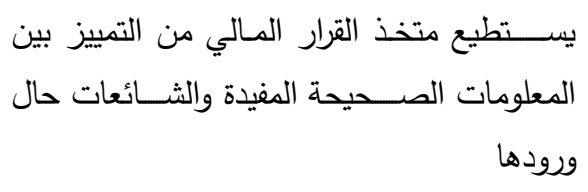 & $\varepsilon$ \\
\hline$\cdot, 1 \cdot v$ & •, V07 & $\varepsilon, r$. & 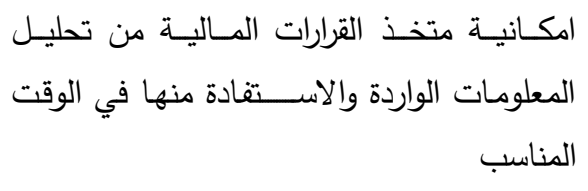 & 。 \\
\hline$\cdot, \bullet \wedge \vee$ & $\cdot, 711$ & $\varepsilon, 17$ & تسـاسبة او اكثر منافسة المالية باتخاذ قرارات تسـعيرية & 7 \\
\hline$\cdot, 11 r$ & $\cdot, \vee \vee$. & r,vA & توالنقدية بثكل مناسك المهارات على الموازنة بين المديونية & V \\
\hline$\cdot, 1 \ldots$ & $\cdot, V \cdot V$ & $\varepsilon, 1$. & 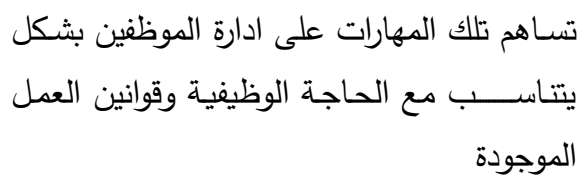 & $\wedge$ \\
\hline$\cdot, 1 \cdot v$ & $\cdot, \vee 04$ & $r, \wedge$. & تقوم تلك المهارات بتحفيز الابداع عند تســــيل & 9 \\
\hline$\cdot, 117$ & $\cdot, \wedge \backslash \wedge$ & $r, q r$ & 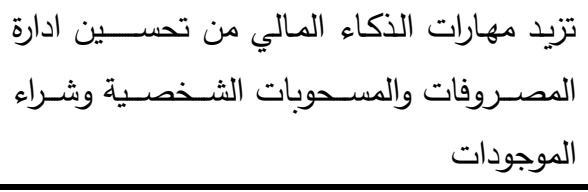 & 1. \\
\hline 0.0982 & $\cdot, 7 \leq 9$ & $r, q q$. & المتوسط العام & \\
\hline
\end{tabular}

المحور الرابع- ت تأثير مؤهلات الذكاء المالي على تنمية سوق الاورلق المالية

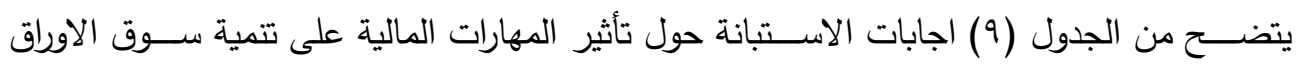

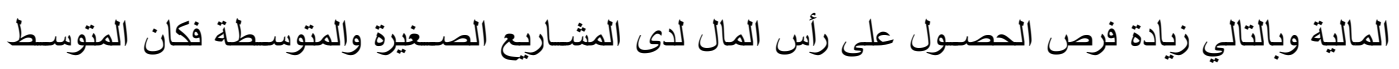




\section{أثر مؤهلات الذكاء المالي على التنمية الرأسمالية. الروازق كاظم والعامري}

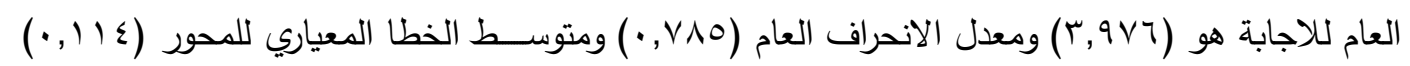
حيث ان الاتجاه العام للاجابة هو موافق، وهي دلالة على مدى تأثير تلك المهارات في زيادة كفاءة السـوق،

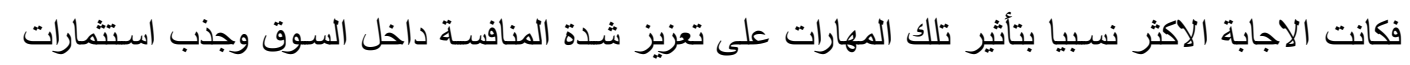
اضافية اخرى محلية داخل السوق بنسبة (ع (,ع) تليها فقرة تعزيز روح المنافسة في تتمية المشاريع الناشئة

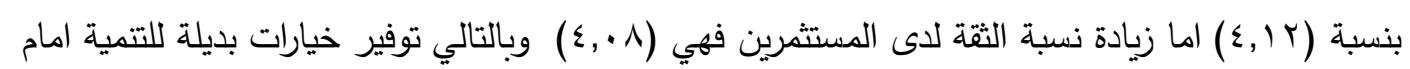
المشاريع الناشئة.

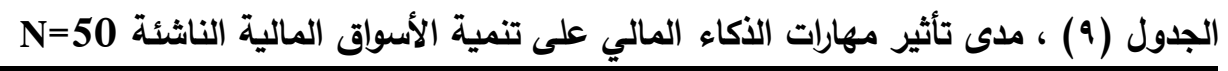

\begin{tabular}{|c|c|c|c|c|}
\hline متوسط الخطأ & المعراف & 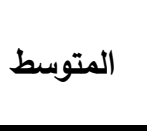 & 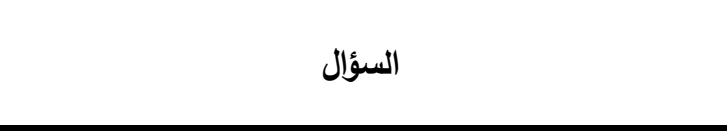 & $ت$ \\
\hline$\cdot, 1 Y 4$ & $\cdot, 9 \cdot$ & $\varepsilon, \cdot \wedge$ & تساعد مؤهلات الذكاء المالي على كسب ثقة المستثرين & 1 \\
\hline$\cdot, 1 \cdot 0$ & $\cdot, \mathrm{V} \varepsilon \cdot$ & r,q & تعوائد رأس مؤهلات المال الذكاء المـالي من تقديم ضــــــانات كافية & r \\
\hline$\cdot, .90$ & $\cdot$, TV. & $\varepsilon, 1 \leqslant$ & تحفتز مؤهلات الذكاء المالي من تعزيز شــــة المنافــــة بين & r \\
\hline 竞 & $\cdot, 9 \mu \mathrm{r}$ & r,v^ & تالأسواق المالية مؤلات الذكاء المالي من تقليل نسبة المخاطر في & $\varepsilon$ \\
\hline$\cdot, 1 Y 9$ & $\cdot, 910$ & r, १ᄉ & شأنها استقطاب على فتح قنوات مالية دولية بين الأسواق المالية من & ० \\
\hline$\cdot, .90$ & $\cdot$, TV. & $\varepsilon, 1 \varepsilon$ & فتوم بتشجيع أصحاب الاموال المحليين من استثمار أموالهم & 7 \\
\hline$\cdot, 1 \Gamma \cdot$ & $\cdot, 79 \leqslant$ & $r, v \uparrow$ & مما يخلق بيئة مالية مناسبة الاتصالات بين متخذي القرارات المالية & $\mathrm{v}$ \\
\hline$\cdot, .9 V$ & $\cdot, 719$ & $\varepsilon, 1 Y$ & 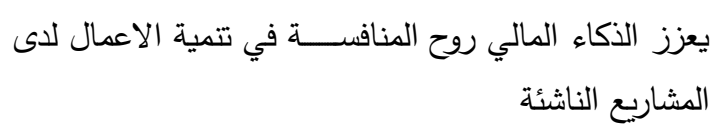 & $\wedge$ \\
\hline$\cdot, 1 \cdot r$ & $\cdot, V Y \wedge$ & $\varepsilon, \cdots$ & من شأنها تقديم تسهيلات لسوق المالي على فتح قنوات مع الجهات الحكومية المالية & 9 \\
\hline$\cdot, 1 \Gamma$ & $\cdot, 919$ & r,Ar & يحفز الذكاء المالي المصـــــارف المحلية على تقديم قروض & 1 . \\
\hline$\cdot, 11 \leq$ & $\cdot, \vee \wedge \diamond$ & 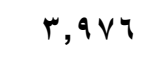 & المتوسط العام & \\
\hline
\end{tabular}

ثالثا: اختبار فرضية البحث

TANMIYAT AL-RAFIDAIN (P-ISSN: 1609-591X; E-ISSN: 2664-276X) تنمية الرافدين 
يعرض الجدول (• ( ) مصـفوفة معامل ارتباط بيرســون بين محاور الاســتبانة، فكان الارتباط بين

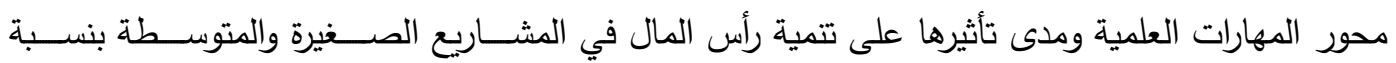

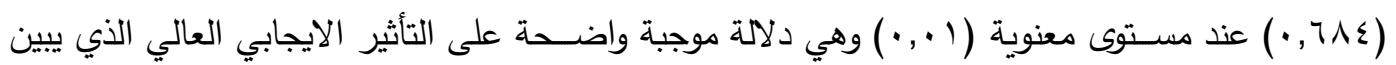

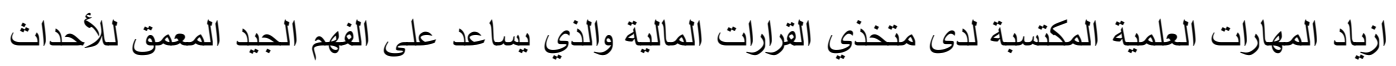

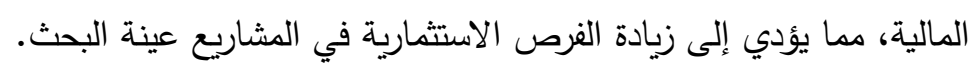
أما الارتباط بين محور المهارات الفنية لدى متخذي القرارات المالية والتتمية الرأسمالية للمشاريع عينة

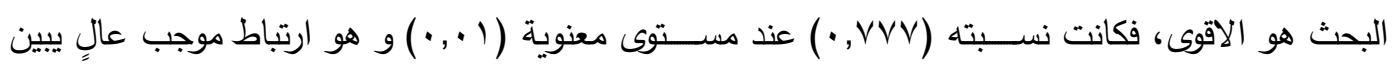

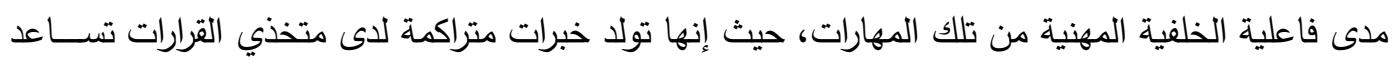
في القراءة الدقيقة للارقام الموجودة في بيئة السوق وبالتالي الحصول من على السعر العادل للسهر. كذلك ارتباط بين محور المهارات العلمية (المحور الاول) ومحور المهارات الفنية ( المحور الثاني)

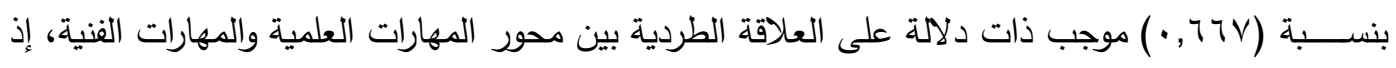

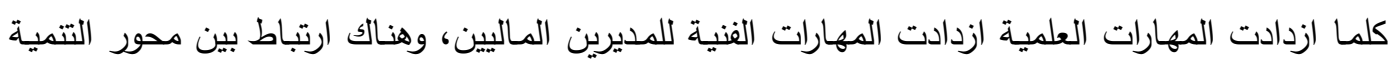

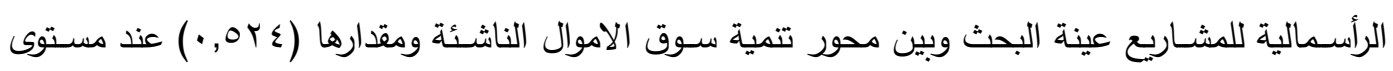
دلالة ( ا ., ·) وهو ارتباط موجب قوي يظهر الأهمية النســبية بين التتمية الرأســمالية في المشــاريع عينة البحث وتتمية سوق الاوراق المالية، إذ إن كفاءة السوق تساعد على توفير ما تتطلبه تلك المشاريع من رأس الس اله المال لتتمية اعمالها، وبالتالي فان النمو المستمر في اعمال تلك المشاريع يساعد على استقطاب رأس المال

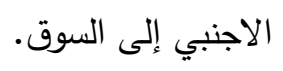
أما الارتباط بين محور المهارات العلمية وتتمية سـوق الاوراق المالية فهو (ب9 ؟ـ • ) عند مسـتوى دلالة ( ( . • ) موجب ذات دلالة واضـحة بان هذه المهارات تتطلب المراجعة المسـتمرة للمعلومات السـوقية

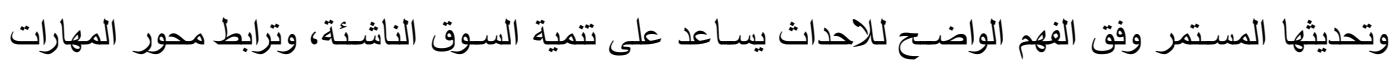

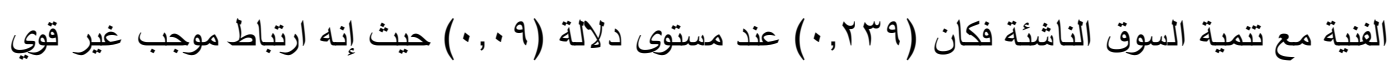

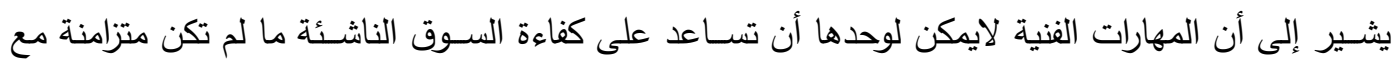
المهارات العلمية.

الجدول (• (1) ،الارتباط النسبي بين محاور الدرسة 


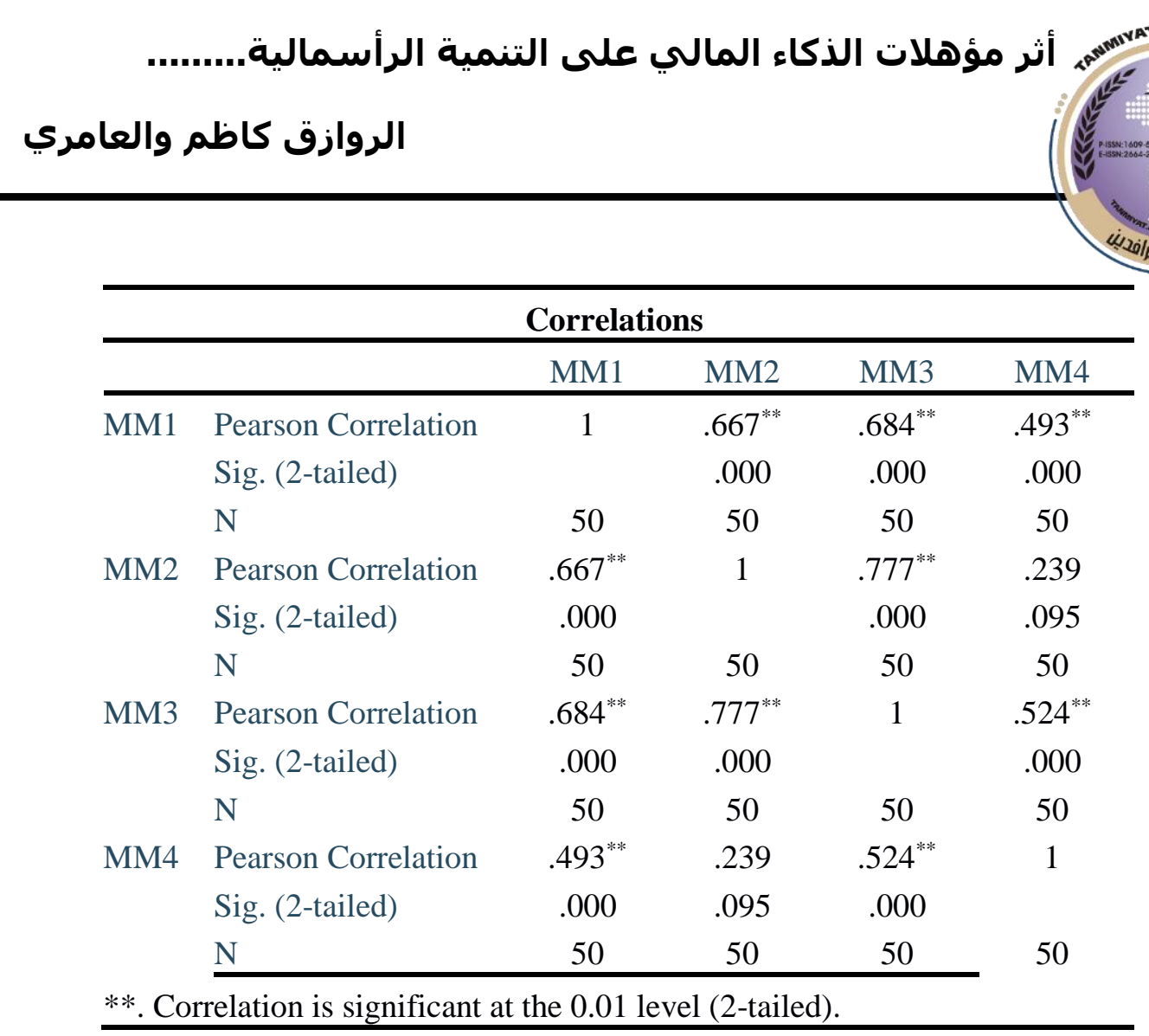

اختبار F test,يظهر الجدول (l) (1) قبول فرضية البحث، حيث تم اختبار F test بمقدار (דrع,. ץ)

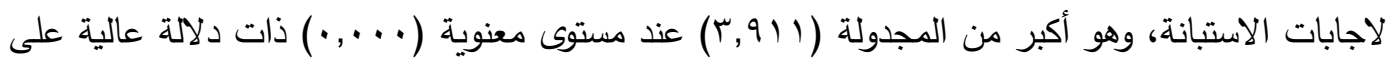

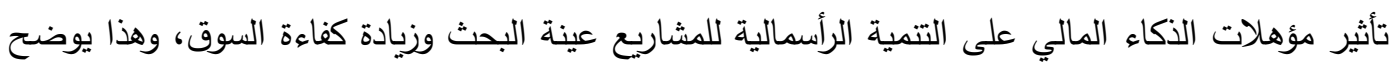
الدور الرئيس لزيادة للمؤهلات العملية والفنية لمتخذي القرارات في زيادة إمكانية التحليل الجيد للمعلومات

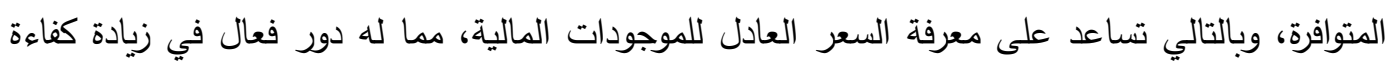

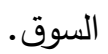

\begin{tabular}{lccccc} 
& & \multicolumn{4}{c}{ ANOVA* (ختبار } \\
\hline \multicolumn{1}{c}{ Model } & $\begin{array}{l}\text { Sum of } \\
\text { Square }\end{array}$ & d.f & $\begin{array}{c}\text { Mean } \\
\text { square }\end{array}$ & F & Sig. \\
\hline Regression & 3.911 & 3 & 1.304 & 20.436 & $.000 * *$ \\
Residual & 2.934 & 46 & 0.64 & & \\
Total & 6.845 & 49 & & & \\
\hline
\end{tabular}

*dependent variable: MMM1

** predictors: ( constant) MMM4, MMM3, MMM3

الاستنتاجات والتوصيات

أولا: الاستتتاجات:

TANMIYAT AL-RAFIDAIN (P-ISSN: 1609-591X; E-ISSN: 2664-276X) تنمية الرافدين 
1- ازدياد الحاجة إلى تتمية مهارات الذكاء المالي في البيئة المالية والذي يسـاعد على خلق فرص التمويل

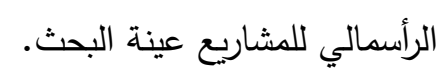

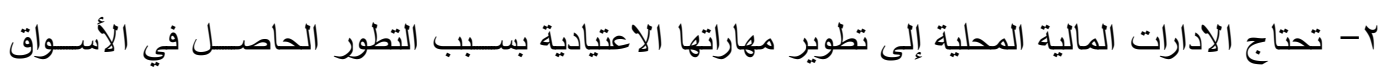

$$
\text { المالية العالمية وشدة التتافس الدولي الرأسمالي. }
$$

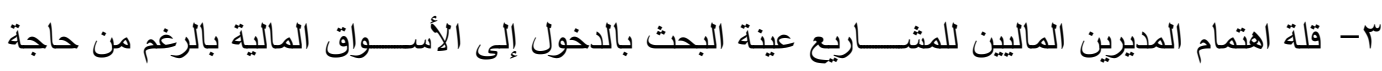

$$
\text { مشاريعهم إلى تتمية رأس المال. }
$$

ع- عدم وجود اهتمام كافٍ من الجهات الحكومية بتوفير رؤوس الاموال اللازمة للمشـــــاريع الصــــيرة

$$
\text { والمتوسطة، وكذلك عدم الاهتمام بتتمية المهارات المالية لتلك المشاريع. }
$$

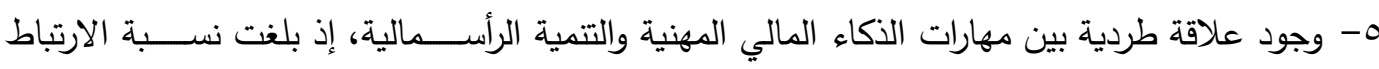

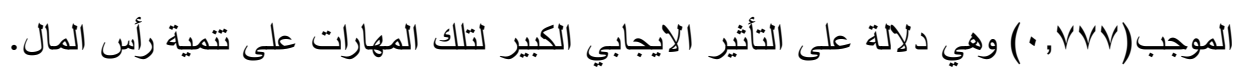

$$
\text { ثانياً: التوصيات }
$$

1- ضرورة تنظيم ورش عمل تضم كل من مديري المشاريع مع الخبراء الماليين تساعد على تتمية المهارات

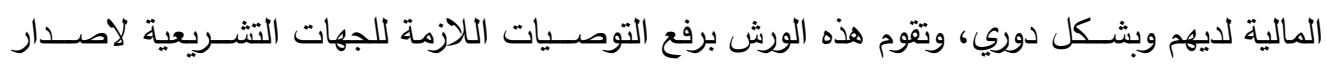

$$
\text { قوانين من شأنها تسهيل وتتظيم عمل الأسواق المالية العراقية. }
$$

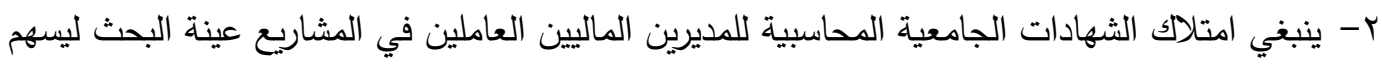

$$
\text { في إمكانية تتمية مهارات الذكاء المالي لديهم. }
$$

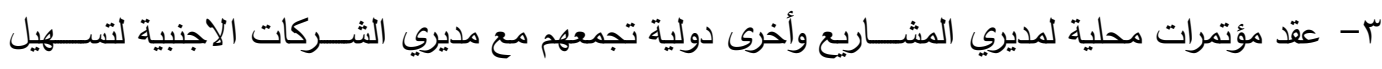

$$
\text { اندماج المشاريع العراقية مع الاجنبية وتداخل أعمالها. }
$$

ع- تتظيم سوق العراق للاوراق المالية بما يتناسب مع التتافس الدولي في عملية كسب الاستثمار الاجنبي

ه- ينبغي اعتماد التطبيقات المحاسـبية والتدقيقية قانونيا بدلا من السـجلات التقليدية كونها تسهم في دقة

$$
\text { وسرعة العمل المحاسبي، وبالتالي تدعم اتخاذ القرارات المالية المفاجأة. }
$$

\section{Reference:-}

Alaaridhy J., Aljiboree A., (2015), Financial Engineering and Its Derivative Tools, Theoretical and Applied Concepts, Dar Al-Manhijiah for Publishing and Distribution, Amman, Jordan, $1^{\text {st }}$ Edition.

Alhamzawee M., (2000), Economics of Banking Credit, Knowledge Foundation, 2nd Edition, Alexandria, Egypt.

Alnasih A., \& Kamil H., (2008), The reality of small and medium industries in Iraq and their impact on employment, Journal of Administration and Economics, No. 69.

Alward A., \& Abdulraheem R., (2006), A proposed strategy for the development of small and medium enterprises in Iraq, Journal of Baghdad College of Economic Sciences Universal, No. 12.

Bettal A., Alrawee M., \& Ali W.,(2011), The role of private banks in financing small and medium enterprises in Iraq, Anbar University Journal for Economic and Administrative Sciences, Volume 4, Issue 7. 


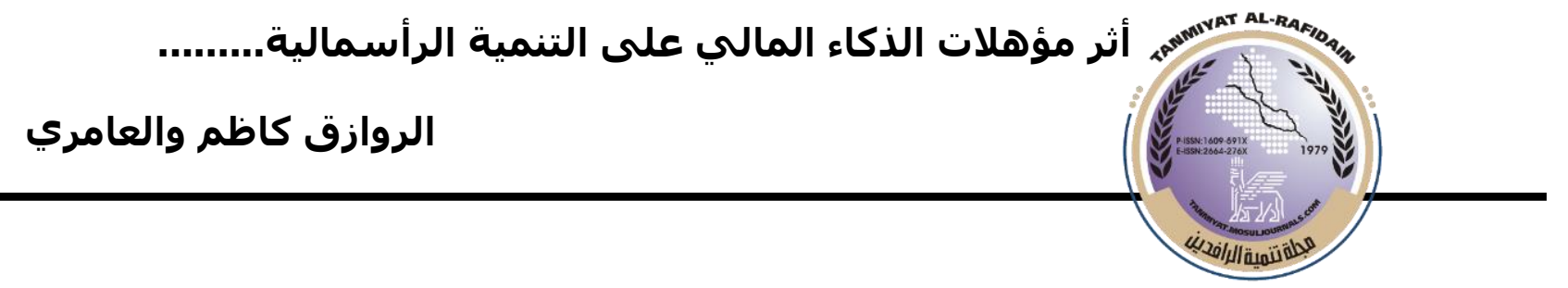

Bsharst H., (2008), Islamic Banking Financing for Small and Medium Enterprises, Dar AlNafees, Amman, Jordan.

Berman, K., Knight, J. and Case, J. (201r), Financial Intelligence, A managers Guide to Knowing What the Numbers Really Mean, 2nd edition.

Chang, M. (2015). Starting your company successfully: From the money perspective. IEEE Engineering Management Review, 43(3).

Didan S., (2016), The reality of small and medium enterprises in Algeria, Master Thesis submitted to the Department of Economic Sciences at Abu Bakr Belkaid, University, Algeria.

Ibraheem,A., \& Alrawee A., (2014), Financial intelligence between the argument of the analyst's qualifications and the emerging financial market efficiency, Jordan as a model, Tenmiettalrafidain, No. 115.

Julie P., Bryn S. and Irene G.(2013), financial intelligence a guide to for social enterprise, the Demonstrating Value Initiative, www.demonstratingvalue.org.

Kristen, B., Karim, E., Harrison, H., Thomas, S., and Joe, T., (2016). Corporate financial Intelligence: Bridging the Educational Gap, WIP.

Schutte, Maria, and Unlu, Emre, (2009), Do Security Analysts reduce Noise, Financial Analysts Journal, Vol. 65 , No.3, May- June.

Samkin, G., Pitu, A. \& Low, M., (2014), Identifying the Financial Literacy Skills Necessary to Run a Small New Zealand Business, e-Journal of Business Education \& Scholarship of Teaching, Vol. 8, Iss. 1, 2014.

Spiech, Steve ,(2005), Ho to be a Great Financial Analyst, It takes seven key skills and a new way of thinking, Journal of Strategic Finance, Vol. 86, Issue 10, April .

Scott, M., \& Bruce, R. (1987). Five stages of growth in small business. Long Range Planning, 20(3).

Sekhree A., (1995), Principles of unitary economics, Office of University Press, Algeria.

Vohora, A., Wright, M., \& Lockett, A. (2004). Critical junctures in the development of university high-tech spinout companies. Research Policy, 33(1).

TANMIYAT AL-RAFIDAIN (P-ISSN: 1609-591X; E-ISSN: 2664-276X) تنمية الرافدين 